\title{
ON THE STRUCTURE OF SOLUTIONS TO THE STATIC VACUUM EINSTEIN EQUATIONS
}

\author{
MICHAEL T. ANDERSON
}

\section{INTRODUCTION}

The static vacuum Einstein equations are the equations

$$
\begin{gathered}
u r=D^{2} u, \\
\Delta u=0,
\end{gathered}
$$

on a Riemannian 3-manifold $(M, g)$, with $u$ a positive function on $M$. Here $r$ denotes the Ricci curvature, $D^{2}$ the Hessian, and $\Delta=\operatorname{tr} D^{2}$ the Laplacian on $(M, g)$. Solutions of these equations define a Ricci-flat 4-manifold $N$, of the form $N=M \times_{u} S^{1}$ or $N=M \times_{u} \mathbb{R}$, with Riemannian or Lorentzian metric of the form

$$
g_{N}=g_{M} \pm u^{2} d t^{2} .
$$

These equations are the simplest equations for Ricci-flat 4-manifolds. They have been extensively studied in the physics literature on classical relativity, where the solutions represent space-times outside regions of matter which are translation and reflection invariant in the time direction $t$. However, with the exception of some notable instances, (c.f. Theorem 0.1 below), many of the global properties of solutions have not been rigorously examined, either from mathematical or physical points of view, c.f. $[\mathrm{Br}]$ for example.

This paper is also motivated by the fact that solutions of the static vacuum equations arise in the study of degenerations of Yamabe metrics (or metrics of constant scalar curvature) on 3-manifolds, c.f. [A1]. Because of this and other related applications of these equations to the geometry of 3-manifolds, we are interested in general mathematical aspects of the equations and their solutions which might not be physically relevant; for example, we allow solutions with negative mass.

In this paper, we will be mostly concerned with the geometry of the 3 -manifold solutions $(M, g, u)$ of (0.1), (i.e. the space-like hypersurfaces), and not with the 4-manifold metric. Thus, the choice of Riemannian or Lorentzian geometry on $N$ in $(0.2)$ will play no role. This considerably simplifies the discussion of singularities and boundary structure, but still allows for a large variety of behaviors; c.f. $[\mathrm{ES}]$ for a survey on singularities of space-times.

Obviously, there are no non-flat solutions to (0.1) on closed manifolds, and so it will be assumed that $M$ is an open, connected oriented 3-manifold. Let $\bar{M}$ be the metric (or Cauchy) completion of $M$ and $\partial M$ the metric boundary, so that $\bar{M}=M \cup \partial M$ is complete as a metric space.

In order to avoid trivial ambiguities, we will only consider maximal solutions of the equations (0.1). For example any domain $\Omega$ in $\mathbb{R}^{3}$ with the flat metric, and $u$ a positive constant, satisfies (0.1). In this case, the metric boundary $\partial \Omega$ is artificial, and has no intrinsic relation with the geometry of the solution. The solution obviously extends to a larger domain, i.e. $\mathbb{R}^{3}$. Thus, we only consider maximal solutions $(M, g, u)$, in the sense that $(M, g, u)$ does not extend to a larger domain $\left(M^{\prime}, g^{\prime}\right) \supset(M, g)$ with $u>0$ on $M^{\prime}$. It follows that at the metric boundary $\partial M$ of $M$, either the metric or $u$ degenerates in some way or $u$ approaches 0 in some way, (or a combination of such).

Partially supported by NSF Grants DMS 9505744 and 9802722. 
A classical result of Lichnerowicz [L1, p.137] implies that if the metric $(M, g)$ satisfying $(0.1)$ is complete, (i.e. $\partial M=\emptyset$ ), and $u \rightarrow 1$ at infinity, then $u \equiv 1$ and $M$ is flat, i.e. $\mathbb{R}^{3}$ or a quotient of $\mathbb{R}^{3}$. More generally, it is proved in [A1, Thm.3.2] that if $(M, g)$ is a complete solution to (0.1), (hence $u>0$ everywhere), then $(M, g)$ is flat and $u$ is constant, i.e. the assumption on the asymptotic behavior of $u$ is not necessary, c.f. also Theorem 1.1 below.

Thus, there are no complete non-trivial solutions to $(0.1)$ and hence $\partial M$ must be non-empty. The set formally given by

$$
\Sigma=\{u=0\} \subset \bar{M},
$$

is called the horizon. It is closely related to the notion of event horizon in general relativity. More precisely, $\Sigma$ may be defined as the set of limit points of Cauchy sequences on $(M, g)$ on which $u$ converges to 0 . Although $\partial M \neq \emptyset$, it is possible that $\Sigma=\emptyset$. However, most solutions of physical interest do have $\Sigma \neq \emptyset$. In the framework of classical relativity, the non-triviality of a static vacuum solution, i.e. the non-vanishing of its curvature, is due to the presence of matter or field sources at $\partial M$, or 'inside' the horizon $\Sigma$ in case $(M, g)$ extends as a vacuum solution past $\Sigma$.

It is natural to consider the situation where $(M, g)$ is not complete and for which the metric boundary $\partial M$ of $M$ coincides with the horizon $\Sigma$. More precisely, we will say that $(M, g)$ is complete away from the horizon $\Sigma$ if for any sequence $p_{i} \rightarrow p \in \partial M$ in the metric topology on $\bar{M}=M \cup \partial M$ one has $u\left(p_{i}\right) \rightarrow 0$. Conversely if $\left\{p_{i}\right\}$ is a bounded sequence in $M$ with $u\left(p_{i}\right) \rightarrow$ 0 , then the definition of $(M, g, u)$ implies that a subsequence of $\left\{p_{i}\right\}$ converges to a point $p \in \partial M$. Thus, $\partial M=\Sigma$ is given by the Hausdorff limit of the $\varepsilon$-levels $L^{\varepsilon}$ of $u$ as $\varepsilon \rightarrow 0$.

While most solutions $(M, g)$ of physical interest are complete away from $\Sigma$, there are many solutions for which this is not the case, c.f. $\S 2$ for further discussion. In such examples, the curvature typically blows up within a finite distance to $\Sigma$. Among the solutions which are complete away from $\Sigma$, most all are singular at $\Sigma$, again in the sense that the curvature of the metric $g$ blows up on approach to $\Sigma$. This is closely related to the fact that the equations (0.1) are formally degenerate at $\Sigma$. Thus, in general, even when $M$ is complete away from $\Sigma$, the metric completion $M \cup \Sigma$ need not be a smooth manifold with boundary.

We will say that $(M, g, u)$ extends smoothly to $\Sigma$, if (i): the set $\Sigma$ is a smooth surface and the partial completion $M^{\prime}=M \cup \Sigma \subset \bar{M}$ is a smooth manifold with boundary, (ii): the metric $g$ extends smoothly to a smooth Riemannian metric on $M^{\prime}$, and (iii): the potential $u$ extends smoothly to $\Sigma$ with $\Sigma=\{u=0\}$; smoothness here means at least $C^{2}$. Note that it might be possible that $\Sigma$ has infinitely many components, or non-compact components of infinite topological type. In any case, one immediate strong consequence of $(0.1)$ is that if $g$ extends smoothly to $\Sigma$, then at $\Sigma$ one has

$$
\left.D^{2} u\right|_{\Sigma}=0 \text {. }
$$

Thus, $\Sigma$ is a totally geodesic surface in $\bar{M}$ and $|\nabla u|$ is a non-zero constant on each component of $\Sigma$, c.f. Remark 1.5 below. Observe that if $(M, g)$ is smooth up to $\Sigma$, and complete away from $\Sigma$, then the isometric double of $M$ across $\Sigma$ is a smooth complete Riemannian manifold. The harmonic function $u$ extends smoothly across $\Sigma$ as harmonic function, odd w.r.t. reflection in $\Sigma$.

By far the most significant solution of the static vacuum equations is the Schwarzschild metric, of mass $m$, given on the space-like hypersurface $M$ by

$$
g_{S}=\left(1-\frac{2 m}{r}\right)^{-1} d r^{2}+r^{2} d s_{S^{2}}^{2}, u=\left(1-\frac{2 m}{r}\right)^{1 / 2}, r>2 m .
$$

This metric models the vacuum exterior region of an isolated static star or black hole. It is a spherically symmetric metric on $M=(2 m, \infty) \times S^{2}$ and has $\Sigma$ given by a (totally geodesic) symmetric $S^{2}$, of radius $2 m$. The mass $m$ is usually assumed to be positive, but we will not make this assumption here. Thus, we allow $m \leq 0$. Of course if $m=0$, then $g_{S}$ is just the flat metric with $u=1$. If $m<0,(0.4)$ is understood to be defined for $r>0$. 
The Schwarzschild metric is asymptotically flat in the sense that there is a compact set $K$ in $M$ such that $M \backslash K$ is diffeomorphic to $\mathbb{R}^{3} \backslash B(R)$, and in a suitable chart on $M \backslash K$, the metric approaches the Euclidean metric at a rate of $1 / r$, i.e.

$$
g_{i j}=\left(1+\frac{2 m}{r}\right) \delta_{i j}+O\left(1 / r^{2}\right),
$$

with curvature decay of order $1 / r^{3}, r=|x|$, and with $m \in \mathbb{R}$. The function $u$ (up to a multiplicative constant) has the asymptotic form $u=1-\frac{m}{r}+O\left(r^{-2}\right)$ with $|\nabla u|=O\left(1 / r^{2}\right)$. A triple $(M, g, u)$ satisfying these conditions is called asymptotically flat.

We note the following remarkable characterization of the Schwarzschild metric.

Theorem 0.1. (Black-hole uniqueness),[I1],[Ro],[BM]

Let $(M, g, u)$ be a solution of the static vacuum Einstein equations, which is smooth up to $\Sigma$ and complete away from $\Sigma$. If $\Sigma$ is a compact, (possibly disconnected) surface and $(M, g, u)$ is asymptotically flat, then $(M, g, u)$ is the Schwarzschild metric, with $m>0$.

The hypothesis that the space-like hypersurface $(M, g, u)$ is asymptotically flat is very common in physics. Namely, in modeling a static space-time outside an isolated, i.e. compact, field or matter source, it is natural to assume that in regions far away from the source the geometry of space approximates that of $\mathbb{R}^{3}$, i.e. empty space.

Nevertheless, mathematically the asymptotically flat assumption is quite strong in that it severely restricts both the topology and geometry of $(M, g)$ outside a large compact set. Further, the physical reasoning above presupposes that there are no complete non-flat solutions to the vacuum equations (0.1), i.e. that a static gravitational field is non-empty solely due the presence of matter somewhere.

Remark 0.2. This latter issue in fact led Einstein to hypothesize that space $M$ is compact, in order to avoid dealing with 'artificial' boundary value problems at infinity, c.f. [E, p.98ff.]. This issue, closely related to Mach's Principle, is discussed in some detail in work of Lichnerowicz, (Propositions A and $B$ of [L1, §31] and [L2, Ch.II]); c.f. also [MTW, §21.12,],[Ri, §9.12] for instance.

As remarked above, there are in fact no complete non-trivial static vacuum solutions, so that the asymptotically flat assumption in Theorem 0.1 may not be unreasonable, c.f. however [El], [G]. In fact, the main result of this paper is that this hypothesis is not necessary in most circumstances; it follows from much weaker assumptions.

To explain this, we first need to consider a weakening of the condition that $\partial M$ is compact. Let

$$
t(x)=\operatorname{dist}(x, \partial M) .
$$

Define $\partial M$ to be pseudo-compact if there is a tubular neighborhood $U$ of $\partial M$ whose boundary $\partial U$ in $M, \partial U \cap M$, is compact, i.e. $\left\{t(x)=s_{o}\right\}$ is compact, for some $s_{o}>0$, (and hence all $\left.0<s_{o}<\infty\right)$. As will be seen in $\S 2$, there are numerous examples of static vacuum solutions with $\partial M$ pseudo-compact but not compact.

Let $E$ be an end, i.e. an unbounded component, of $\bar{M} \backslash U$. The mass of $E$ may be defined by

$$
m_{E}=\lim _{s \rightarrow \infty} m_{E}(s)=\lim _{s \rightarrow \infty} \frac{1}{4 \pi} \int_{S_{E}(s)}<\nabla \log u, \nabla t>d A,
$$

where $S_{E}(s)=t^{-1}(s) \cap E$. Since $u$ is harmonic, $\log u$ is superharmonic, so that the divergence theorem implies that $m_{E}(s)$ is monotone decreasing in $s$. Hence the limit (0.7) is well-defined, (possibly $-\infty$ ). Note that the static vacuum equations are invariant under multiplication of $u$ by positive constants. We use the logu term in (0.7) in place of $u$ so that the mass is independent of this rescaling in $u$.

The following is the main result of this paper. 
Theorem 0.3. Let $(M, g, u)$ be a solution of the static vacuum Einstein equations.

(i). Suppose $\partial M$ is pseudo-compact.

Then $\bar{M} \backslash U$ has only finitely many ends $\left\{E_{i}\right\}, 0 \leq i \leq q<\infty$. Supposing $q>0$ and (i) holds, let $E \in\left\{E_{i}\right\}$ be any end of $M$ satisfying

(ii). $u\left(x_{i}\right) \geq u_{o}$, for some constant $u_{o}>0$ and some sequence $x_{i} \in E$ with $t\left(x_{i}\right) \rightarrow \infty$.

Then the end $(E, g, u)$ is either asymptotically flat, or small, in the sense that the area growth of geodesic spheres satisfies

$$
\int^{\infty} \frac{1}{\operatorname{areaS_{E}(s)}} d s=\infty
$$

Further, if $m_{E} \neq 0$ and $\sup _{E} u<\infty$, then the end $E$ is asymptotically flat.

We make several remarks on this result. First, if $\partial M$ is not pseudo-compact, then it is easy to construct static vacuum solutions for which $\bar{M} \backslash U$ has infinitely many ends, c.f. the end of $\S 1$ or $\S 2(\mathrm{I})$. Further, there are examples of ends $E$ with compact boundary, on which (ii) does not hold and which are neither asymptotically flat nor small in the sense of (0.8), c.f. Example 2.11. Thus, both hypotheses (i) and (ii) are necessary in Theorem 0.3 . There are also examples with $\bar{M}$ compact, c.f. $\S 2(\mathrm{I})$, so that $\bar{M} \backslash U$ may have no ends, $(q=0)$.

On the other hand, both alternatives in Theorem 0.3, namely asymptotically flat or small ends, do occur. Asymptotically flat ends satisfy area $S_{E}(s) \sim 4 \pi s^{2}$ as $s \rightarrow \infty$, while small ends have small area growth. For example, (0.8) implies, at least, that there is a sequence $s_{i} \rightarrow \infty$ such that

$$
\operatorname{area} S_{E}\left(s_{i}\right) \leq s_{i} \cdot\left(\log s_{i}\right)^{1+\varepsilon},
$$

for any fixed $\varepsilon>0$. All known examples of solutions satisfying (0.8) are topologically of the form $\left(\mathbb{R}^{2} \backslash B\right) \times S^{1}$ outside a compact set and the geodesic spheres have at most linear area growth. The main example of a static vacuum solution with a small end is the family of Kasner metrics, c.f. Example 2.11 below.

To illustrate the sharpness of the last statement in Theorem 0.3 , we construct in Remark 3.8 a (dipole-type) static vacuum solution $(M, g, u)$ with a single end $E$ on which $m_{E}=0, \sup _{E}<\infty$, and which satisfies (0.8). Similarly, Example 2.11 provides static vacuum solutions with $m_{E}>0$, $\sup _{E} u=\infty$ and satisfying (0.8). Thus, the last result is also sharp.

Assuming $\partial M$ is pseudo-compact, it is easy to see that if $m_{E}>0$ then (ii) holds, so that (ii) may be replaced by the assumption $m_{E}>0$. Thus, for the physically very reasonable class of solutions such that $\partial M$ is pseudo-compact, $m_{E}>0$ for all ends $E$, and $u$ is bounded, all ends $E$ of $M$ are asymptotically flat. We also point out that if $M$ is complete away from $\Sigma$, then (ii) holds at least on some end $E$.

The proof of Theorem 0.3 gives some further information on the asymptotic structure of the small ends. For instance, the curvature in such ends decays at least quadratically, c.f. (1.3), and in the geodesic annuli $A_{E}\left(\frac{1}{2} s_{i}, 2 s_{i}\right)$ in $E$, the metric approaches in a natural sense that of a Weyl solution, i.e. a static axisymmetric solution, as $s_{i} \rightarrow \infty$. Thus, asymptotically, small ends have at least one non-trivial Killing field. However, it is not known for example if the metric is asymptotic to a unique Weyl solution, or even if small ends are necessarily of finite topological type.

It would also be of interest to prove that $(M, g, u)$ has a unique end if $\partial M$ is pseudo-compact. However, we have not been able to do this without further assumptions, c.f. Remark 3.10.

The definition of asymptotically flat rules out the possibility that $M$ is smooth up to $\Sigma$ and complete away from $\Sigma$, with $\Sigma$ non-compact, since for example $u=0$ on $\Sigma$ while $u \rightarrow 1$ on any asymptotically flat end. Consider however the following (B1) solution,

$$
g_{B 1}=\left(1-\frac{2 m}{r}\right)^{-1} d r^{2}+\left(1-\frac{2 m}{r}\right) d \phi^{2}+r^{2} d \theta^{2},
$$


where $\phi / 4 m \in[0,2 \pi], \theta \in[0, \pi], r \geq 2 m>0$, with $u=r \cdot \sin (\theta)$. Note that the potential $u$ is unbounded.

The B1 metric is just a 3-dimensional slice of the 4-dimensional Schwarzschild metric $\left(N, g_{S}+\right.$ $\left.u^{2} d \theta^{2}\right),\left(t\right.$ changed to $\theta$ in (0.2)), obtained by dividing $N$ by an $S^{1} \subset \operatorname{Isom}\left(S^{2}\right)$ orthogonal to $d \theta$; this is the slice 'orthogonal' to the usual slice giving the Schwarzschild metric (0.4). This metric has $\Sigma$ given by two disjoint, isometric copies of $\mathbb{R}^{2}$, each of positive Gauss curvature and asymptotic to a flat cylinder. It is smooth up to $\Sigma$ and complete away from $\Sigma$. The metric is globally asymptotic to the flat metric on $\mathbb{R}^{2} \times S^{1}$, again with curvature decay of order $O\left(1 / r^{3}\right)$ and with $u$ of linear growth in distance to $\Sigma$. Such solutions will be called asymptotically cylindrical. In fact a large class of Weyl solutions have 'dual' solutions in this sense which are asymptotically cylindrical, c.f. Remark 2.9.

This paper is organized as follows. Following discussion of some general topics on static spacetimes in $\S 1$, we analyse in some detail the class of Weyl vacuum solutions in $\S 2$. Several new results on the structure of these solutions are given; for instance Proposition 2.2 gives a new characterization of Weyl metrics. In addition, some efforts have been made to give a reasonably clear and organized account of the breadth of possibilities and behavior of Weyl metrics, since their treatment in the literature is rather sketchy and since they serve as a large class of models on which to test Theorem 0.3 . Theorem 0.3 is proved in $\S 3$, and the paper concludes with several remarks on generalizations, and some open questions.

I would like to thank the referee for suggesting a number of improvements in the exposition of the paper.

\section{Background Discussion.}

Let $(M, g, u)$ be an open, connected oriented Riemannian 3-manifold and $N=M \times_{u} \mathbb{R}$ or $N=M \times{ }_{u} S^{1}$, as in (0.2). Thus $N$ represents a static space-time and $M \subset N$ is totally geodesic. The Einstein field equations on $N$ are

$$
r_{N}-\frac{s_{N}}{2} \cdot g_{N}=T
$$

where $T$ is the ( $\mathbb{R}$ or $S^{1}$-invariant) stress-energy tensor. (We are ignoring physical constants here). These equations may be expressed on the space-like hypersurface $M$ as the system

$$
\begin{gathered}
r-u^{-1} D^{2} u+\left(u^{-1} \Delta u-\frac{1}{2} s\right) \cdot g=T_{H}, \\
\frac{1}{2} s=T_{V},
\end{gathered}
$$

where $T_{H}$ is the horizontal or space-like part of $T$ and $T_{V}$ is the vertical or time-like part of $T$. These are the equations on $M$ for a Lorentzian space-time $N$; in case $N$ is Riemannian, the first equation is the same while the second is $-\frac{1}{2} s=T_{V}$. When $T=0$, one obtains the vacuum equations (0.1), which are the same for Lorentzian or Riemannian signature. A common example, with $T \neq 0$ is a static perfect fluid, c.f. [Wd, Ch.4], with $T$ given by

$$
T=(\mu+\rho) d t^{2}+\rho g,
$$

where $\mu, \rho$ are time independent scalar fields representing the energy density and pressure respectively. The equations (1.2) imply that the full Riemann curvature $R_{N}$ of $N$ is determined by $r, u$ and $T$.

The horizon $\Sigma=\{u=0\}$ corresponds formally to the fixed point set of the $S^{1}$ action on $N$ and requires special consideration. For example, even if $M$ is smooth up to $\Sigma$ the Riemannian 4manifold $\left(N, g_{N}\right)$ might not be smooth across $\Sigma$, even though the curvature $R_{N}$ is smooth. Namely, assuming the $S^{1}$ parameter $t \in[0,2 \pi)$, if $|\nabla u|_{\Sigma} \neq 1$, then $N$ has cone singularities (with constant angle by (0.3)) along and normal to the totally geodesic submanifold $\Sigma \subset N$. (This issue does not 
arise for Lorentzian metrics). By multiplying the potential function $u$ by a suitable constant, one can make the metric $g_{N}$ smooth across any given component of $\Sigma$; one cannot expect however in general that this can be done simultaneously for all components of $\Sigma$, if there are more than one. This issue will reappear in $\S 2$.

The following result is proved in [A1, Cor.A.3]. It implies, (by letting $t \rightarrow \infty$ ), that if $(M, g, u)$ is a complete solution to the static vacuum equations with $u>0$ everywhere, then $M$ is flat, and $u$ is constant.

Theorem 1.1. Let $(M, g, u)$ be a solution to static vacuum equations (0.1). Let $t(x)=\operatorname{dist}(x$, $\partial M)$ as in (0.6). Then there is a constant $K<\infty$, independent of ( $M, g, u)$, such that

$$
|r|(x) \leq \frac{K}{t(x)^{2}},|\nabla \log u|(x) \leq \frac{K}{t(x)} .
$$

Remark 1.2. The same result has recently been proved for stationary vacuum solutions, i.e. spacetimes admitting a complete time-like Killing field, c.f. [A2]. We will discuss elsewhere to what extent Theorem 0.3 generalizes to stationary vacuum solutions.

Recall from $\S 0$ that the potential function $u$ of a static vacuum solution may be freely renormalized by arbitrary positive constants; hence the appearance of $\log u$ in (1.3), as in (0.7).

Theorem 1.1 implies that the curvature of $(M, g)$ is controlled away from $\partial M$, and hence the local geometry of solutions is controlled away from $\partial M$ by lower bounds on the local volume or injectivity radius. More precisely, we have the following results which are essentially a standard application of the Cheeger-Gromov theory of convergence/collapse of Riemannian manifolds, c.f. [CG], $[\mathrm{A} 3, \S 2]$, or also [P, Ch.12] for an introduction to these results. Further details of the proofs of these results are given in [A2], (for the more general class of stationary space-times), and also in [A1,App.].

Lemma 1.3. (Non-Collapse). Let $\left(M_{i}, g_{i}, u_{i}\right)$ be a sequence of solutions to the static vacuum equations (0.1). Suppose

$$
\left|r_{i}\right| \leq \Lambda, \operatorname{diam} M_{i} \leq D, \operatorname{vol} M_{i} \geq \nu_{o}
$$

and

$$
\operatorname{dist}\left(x_{i}, \partial M_{i}\right) \geq \delta
$$

for some $x_{i} \in M_{i}$ and positive constants $\nu_{o}, \Lambda, D, \delta$. Assume also that $u_{i}$ is normalized so that $u_{i}\left(x_{i}\right)=1$.

Then, for any $\epsilon>0$ sufficiently small, there are domains $U_{i} \subset M_{i}$, with $\epsilon / 2 \leq \operatorname{dist}\left(\partial U_{i}, \partial M_{i}\right) \leq \epsilon$, and $x_{i} \in U_{i}$ such that a subsequence of the Riemannian manifolds $\left(U_{i}, g_{i}\right)$ converges, in the $C^{\infty}$ topology, modulo diffeomorphisms, to a limit manifold $(U, g)$, with limit function $u$ and base point $x=\lim x_{i}$. The triple $(U, g, u)$ is a smooth solution of the static equations with $u(x)=1$.

Lemma 1.4. (Collapse). Let $\left(M_{i}, g_{i}, u_{i}\right)$ be a sequence of solutions to the static vacuum equations (0.1). Suppose

$$
\left|r_{i}\right| \leq \Lambda, \operatorname{diam} M_{i} \leq D, \operatorname{vol} M_{i} \rightarrow 0
$$

and

$$
\operatorname{dist}\left(x_{i}, \partial M_{i}\right) \geq \delta
$$

for some $x_{i} \in M_{i}$ and constants $\Lambda, D, \delta$. Assume also that $u_{i}$ is normalized so that $u_{i}\left(x_{i}\right)=1$.

Then, for any $\epsilon>0$ sufficiently small, there are domains $U_{i} \subset M_{i}$, with $\epsilon / 2 \leq \operatorname{dist}\left(\partial U_{i}, \partial M_{i}\right) \leq \epsilon$ with $x_{i} \in U_{i}$, such that $U_{i}$ is either a Seifert fibered space or a torus bundle over an interval. In both cases, the $g_{i}$-diameter of any fiber $F$, (necessarily a circle $S^{1}$ or torus $T^{2}$ ), goes to 0 as $i \rightarrow \infty$, and $\pi_{1}(F)$ injects in $\pi_{1}\left(U_{i}\right)$. 
Consequently, there are infinite $\mathbb{Z}$ or $\mathbb{Z} \oplus \mathbb{Z}$ covers $\widetilde{U}_{i}$ of $U_{i}$, such that $\left\{\widetilde{U}_{i}, g_{i}, x_{i}\right\}$ does not collapse and hence has a subsequence converging smoothly to a limit $(\widetilde{U}, g, x)$ of the static vacuum equations with $x=\lim x_{i}^{\prime}, x_{i}^{\prime}$ a lift of $x_{i}$ to $\widetilde{U}_{i}$. The limit $(\widetilde{U}, g, x)$ admits a free isometric $\mathbb{R}$ or $\mathbb{R} \oplus \mathbb{R}$ action, (c.f. §2), which also leaves the potential function $u$ invariant, and $u(x)=1$.

In studying static solutions, it is often very useful to consider the conformally equivalent metric $\tilde{g}=u^{2} \cdot g$ on $M$. An easy calculation using the behavior of Ricci curvature under conformal deformations, c.f. [Bes, p.59], shows that the Ricci curvature $\tilde{r}$ of $\tilde{g}$, in the vacuum case (0.1), is given by

$$
\tilde{r}=2(d \log u)^{2} \geq 0 .
$$

Further, if $\tilde{\Delta}$ denotes the Laplacian of $\tilde{g}$, then

$$
\tilde{\Delta} \log u=0 .
$$

The equations (1.4)-(1.5) are equivalent to the static vacuum equations (0.1). Since these equations are invariant under the substitution $u \rightarrow-u$, it follows that if $(M, g, u)$ is a static vacuum solution, then so is $\left(M, g^{\prime}, u^{-1}\right)$, with $g^{\prime}$ given by

$$
g^{\prime}=u^{4} \cdot g .
$$

Similarly, observe that if $\left(N, g_{N}\right)$ is the associated Ricci-flat 4-manifold $(0.2)$, then

$$
\Delta_{N} \log u=0 .
$$

Here and below, log always denotes the natural logarithm.

We discuss briefly some of the simplest static vacuum solutions:

\section{Levi-Civita Solutions.}

There are 7 classes of so-called degenerate static vacuum solutions, where the eigenvalues $\lambda_{i}$ of the Ricci curvature $r$ satisfy $\lambda_{1}=\lambda_{2}=-2 \lambda_{3}$, called A1-A3, B1-B3, C, c.f. [EK, §2-3.6]. The $B$ metrics are dual to the $A$ metrics, as mentioned in $\S 0$, c.f. $\S 2$ for details. The A1 metric is the Schwarzschild metric. It is of interest to examine the A2 metric, given in standard cylindrical coordinates on $\mathbb{R}^{3}$ by

$$
g_{A 2}=z^{2}\left(d r^{2}+\left(\sinh ^{2} r\right) d \phi^{2}\right)+\left(\frac{2 m}{z}-1\right)^{-1} d z^{2},
$$

with $u=\left(\frac{2 m}{z}-1\right)^{1 / 2}$ and $z \in[0,2 m], m>0$. The horizon $\Sigma=\{u=0\}$ is given by the set $\{z=2 m\}$ and hence is the complete hyperbolic metric $H^{2}$, with curvature $-(2 m)^{-2}$. It is easily verified that the A2 metric is smooth up to $\Sigma$. However, $\Sigma \neq \partial M$; the set $\{z=0\}$ is at finite distance to $\Sigma$, and so $\partial M$ has another (singular) component obtained by crushing (compact subsets of) the hyperbolic metric to a point.

Let $\Gamma$ be any discrete group of hyperbolic isometries. Then $\Gamma$ extends in an obvious way to a group of isometries of $g_{A 2}$. The uniformization theorem for surfaces implies that any orientable surface except $S^{2}$ and $T^{2}$, including surfaces of infinite topological type and infinitely many ends, admits a complete hyperbolic metric, i.e. is the quotient $H^{2} / \Gamma$, for some $\Gamma$. Hence, any such surface and hyperbolic metric can be realized as the horizon $\Sigma$ of a static vacuum solution.

Topologically, for $\Sigma=H^{2} / \Gamma$, we have $M=\Sigma \times I$. Hence for example if $\Sigma$ has infinitely many ends, then $M$ also has infinitely many ends; in particular, this shows that the hypothesis that $\partial M$ is pseudo-compact in Theorem 0.3 is necessary.

The A3 metric is

$$
g_{A 3}=z^{2}\left(d r^{2}+r^{2} d \phi^{2}\right)+z d z^{2}
$$


with $u=z^{-1 / 2}>0, r>0$. Hence $\Sigma$ is empty in this case - it occurs at infinity in the metric. This metric may be realized as a pointed limit of the A2 metric as $m \rightarrow \infty$ and also as a limit, in a certain sense, of the A1 metric, c.f. Example 2.11.

Remark 1.5. The discussion above raises the natural question if any orientable connected Riemannian surface $(\Sigma, g)$ can be realized as the horizon of a static vacuum solution, smooth up to $\Sigma$, which is defined at least in a neighborhood of $\Sigma$. In general, this appears to be unknown.

Observe that any complete constant curvature metric on an orientable surface can be realized in this way. The Schwarzschild metric gives the constant curvature metric on $S^{2}$, the quotients of the A2 metric give all hyperbolic surfaces, and quotients of the flat metric, with $u$ a linear function, give all flat metrics on a surface, $\left(T^{2}, S^{1} \times \mathbb{R}\right.$, or $\left.\mathbb{R}^{2}\right)$.

Geroch-Hartle show in [GH] that any rotation-invariant metric on $S^{2}$ or $T^{2}$ can be realized at the horizon. Except for the Schwarzschild metric, such solutions are not complete away from $\Sigma$.

Observe that the full 1-jet of $(M, g)$ at $\Sigma$ (assumed connected) is determined solely by the surface metric $(\Sigma, g)$, since $\Sigma$ is totally geodesic and, renormalizing $u$ if necessary, $|\nabla u| \equiv 1$ on $\Sigma$ by (0.3). Observe also that one cannot have $|\nabla u| \equiv 0$ on $\Sigma$, since $u$ is harmonic and the divergence theorem applied to a small neighborhood $U$ of $\Sigma$ would imply that $u \equiv 0$ on $U$, which is ruled out.

On the other hand, the metric $(M, g)$ is not uniquely determined by its boundary values $(\Sigma, g)$. Namely, the flat metric on $T^{2}$ is realized by the flat vacuum solution $M=T^{2} \times \mathbb{R}^{+}, u=t=\operatorname{dist}(\Sigma, \cdot)$ and also (locally) by a non-flat metric, c.f. [T],[P]. Similar remarks hold for local perturbations of the Schwarzschild metric, c.f. [GH].

\section{Weyl Solutions.}

A large and very interesting class of explicit solutions of the static vacuum equations are given by the Weyl solutions [W], c.f. also [EK, $\S 2.3-9]$ or [Kr, Ch.16-18]. In fact, it appears that essentially all known explicit solutions of the static vacuum equations are of this form. Since the literature on these solutions is not very organized or rigorous, especially regarding their global structure, we discuss these solutions in some detail. These metrics will also illustrate the necessity of the hypotheses in Theorem 0.3 .

Definition 2.1. A Weyl solution is a solution $(M, g, u)$ of the static vacuum equations (0.1) which admits an isometric $\mathbb{R}$-action, i.e. a non-zero homomorphism $\mathbb{R} \rightarrow \operatorname{Isom}(M)$, leaving u invariant.

Apriori, the topology of a Weyl solution could be quite non-trivial; for example $M$ could be any Seifert fibered space. The first result shows that only the simplest topology (and geometry) is possible. For the moment, we exclude any possible fixed point set of the $\mathbb{R}$-action from the discussion.

Proposition 2.2. Let $(M, g, u)$ be a Weyl solution with $\mathbb{R}$-action without fixed points, which does not admit a (local) free isometric $\mathbb{R} \times \mathbb{R}$ action. Then the universal cover $(\widetilde{M}, g)$ of $(M, g)$ is a warped product of the form

$$
\widetilde{M}=V \times_{f} \mathbb{R}, g=g_{V}+f^{2} d \phi^{2},
$$

with $\left(V, g_{V}\right)$ a Riemannian surface and $f$ a positive function on $V$. The $\mathbb{R}$-action on $\widetilde{M}$ is by translation on the second factor.

Proof: This result, whose proof is purely local, is a strengthening in this situation of a wellknown result in general relativity, Papapetrou's theorem, c.f. [Wd,Thm.7.1.1], which requires certain global assumptions, (e.g. smoothness up to $\Sigma$ ).

Let $K$ denote the (complete) Killing field generated by the $\mathbb{R}$-action on $M$, and $f=|K|$. We may assume that $u$ is not a constant function on $M$, since if $u$ is constant, the metric is flat, and so 
admits a local $\mathbb{R} \times \mathbb{R}$ action. Since $(M, g, u)$ is real-analytic, $u$ is not constant on any open set in $M$. We thus choose a neighborhood $U$ of any point $p$ where $\nabla u(p) \neq 0$ on which $|\nabla u|>0$. Define $e_{1}$ by $e_{1}=\nabla u /|\nabla u|$, and extend it to a local orthonormal frame $e_{1}, e_{2}, e_{3}$ for which $e_{3}=K /|K|=K / f$. Note that this is possible since $u$ is required to be invariant under the flow of $K$, so that

$$
<\nabla u, K>=0 \text {. }
$$

In $U$, the metric $g$ may be written as

$$
g=\pi^{*} g_{V}+f^{2}(d \phi+\theta)^{2},
$$

where $\pi: U \rightarrow V$ is a Riemannian submersion onto a local surface $\left(V, g_{V}\right), \theta$ is a connection 1-form, $K=\partial / \partial \phi$ and $f$ is a function on the orbit space $V$. If $\theta=0$, then the result follows. Thus, we assume $|\theta|>0$ in $U$ and show this implies that $g$ has a free isometric local $\mathbb{R} \times \mathbb{R}$ action.

Consider the 1-parameter family of metrics

$$
g_{s}=\pi^{*} g_{V}+s^{2} f^{2}\left(d \phi+s^{-2} \theta\right)^{2},
$$

for $s>0$, with $g_{1}=g$. Geometrically, this corresponds to rescaling the length of the fibers of $\pi$ and changing the horizontal distribution of $\pi$, (when $\theta \neq 0)$. Now it is a standard fact that the 1-parameter family of 4-metrics

$$
g_{s}^{4}=g_{s} \pm u^{2} d t^{2}
$$

remains Ricci-flat for all $s$. This can be seen from standard formulas for Riemannian line bundles, c.f. [Bes, 9.36, 9G], [Kr, 16.1-3] or $[\mathrm{A} 2, \S 1.2]$. Thus, the metrics $g_{s}$ all satisfy the static vacuum equations

$$
u r_{s}=D_{s}^{2} u
$$

from (0.1), with the same potential $u$. Equivalently, the conformal metrics $\tilde{g}_{s}=u^{2} g_{s}$ satisfy

$$
\tilde{r}_{s}=2(d \log u)^{2},
$$

for all $s$, c.f. (1.4) The right side of (2.6) is of course independent of $s$.

We claim that the metrics $g_{s}$ on $U$ are all locally isometric. While this could be proved by a lengthy direct computation, we argue more conceptually as follows. Let $e_{i}^{s}$ be a local orthonormal frame for $g_{s}$, determined as above for $g$. We then have $e_{1}^{s}=e_{1}, e_{3}^{s}=s^{-1} e_{3}$ while $e_{2}^{s}$ varies in the plane $\left\langle e_{2}, e_{3}\right\rangle$. The same relations hold w.r.t. $\tilde{g}_{s}$. Recall also that the full curvature tensor is determined by the Ricci curvature in dimension 3. It then follows from these remarks and (2.6) that for each $q \in U$ and $s>0$, there is a sectional curvature preserving isomorphism $F_{s}: T_{q} M \rightarrow T_{q} M$, i.e.

$$
\tilde{K}_{s}\left(F_{s}(P)\right)=\tilde{K}_{1}(P),
$$

where $P$ is any 2-plane and $\tilde{K}_{s}$ is the sectional curvature w.r.t. $\tilde{g}_{s}$. Clearly $F_{s}$ varies smoothly with $q$ and $s$. Using the expression (2.6), a result of Kulkarni $[\mathrm{Ku}]$ then implies that the metrics $\tilde{g}_{s}$ are locally isometric and hence so are the metrics $g_{s}$.

Let $\Omega=d \theta$ be the curvature form of the line bundle $\pi$. Then w.r.t. the metric $g,|\Omega|=$ $\left|\Omega\left(e_{1}, e_{2}\right)\right|=\left|<\nabla_{e_{1}} e_{2}, e_{3}\right\rangle \mid$. The same equalities hold w.r.t. $\Omega_{s}=d \theta_{s}=s^{-2} d \theta$ and the $g_{s}$ metric. A short computation then gives

$$
\left|\Omega_{s}\right|_{g_{s}} \rightarrow 0, \text { as } s \rightarrow \infty \text {. }
$$

Hence consider the behavior of the metrics $g_{s}$ as $s \rightarrow \infty$. We are then expanding or blowing up the metric in the fiber direction, at a given base point. Since the metrics $g_{s}$ are isometric, there are (local) diffeomorphisms $\psi_{s}$ such that $\psi_{s}^{*} g_{s}$ converges to a limit metric $g_{\infty}$. At a given base point, the diffeomorphisms $\psi_{s}$ expand or blow up smaller and smaller intervals of the parameter $\phi$ to unit size, giving rise to a limit parameter $\phi_{\infty}$. When $\theta=0$, this is the only change; the limit metric $g_{\infty}$ is the same as $g$ with the parameter $\phi$ replaced by $\phi_{\infty}$. (This is completely analogous to passing 
from the flat metric on $\mathbb{R}^{2} \backslash\{0\}$ to the flat metric on its universal cover, i.e. unwrapping the circles to lines).

However, when $\theta \neq 0$, the $e_{2}$ direction is also being expanded or blown-up in a similar way. The function $u$ is left invariant under the family $\left\{\psi_{s}\right\}$.

It follows from (2.7) that the limit metric $g_{\infty}$ is a static vacuum solution of the form

$$
g_{\infty}=\pi^{*} g_{V_{\infty}}+f_{\infty}^{2}\left(d \phi_{\infty}\right)^{2},
$$

i.e. the 1 -form $\theta_{\infty}=0$ in the limit. Further, since the $e_{2}$ direction has been blown up, the function $f_{\infty}$ varies only in the $e_{1}$ direction, i.e. $f_{\infty}=f_{\infty}(u)$.

Metrics of the form (2.8) are analysed in detail below. Referring to (2.10), let $r=f_{\infty} \cdot u=h(u)$. In a possibly smaller open subset of $U$, we may invert $h$ and write $u=u(r)$, where $r$ is a local coordinate on $V_{\infty}$. It is easy to see, (c.f. (2.12)-(2.13) below for example), that $g_{\infty}$ admits a nonvanishing Killing field $\partial / \partial z$, tangent to $V_{\infty}$ but orthogonal to $\partial / \partial r$, and hence $g_{\infty}$ admits a free isometric local $\mathbb{R} \times \mathbb{R}$ action. The metrics $g_{s}$ are all locally isometric and so the metric $g=g_{1}$ also has a free isometric local $\mathbb{R} \times \mathbb{R}$ action.

Since the proof above is completely local, Proposition 2.2 holds locally, (in suitably modified form), even if $(M, g)$ admits only a local or partial $\mathbb{R}$-action.

Static vacuum solutions admitting a free isometric local $\mathbb{R} \times \mathbb{R}$ action are completely classified; they are either flat or belong to the family of Kasner metrics, c.f. Example 2.11 below or [EK, Thm.2-3.12]. Such solutions $d o$ have Killing fields $K$ which are not hypersurface orthogonal, i.e. $d \theta \neq 0$ in (2.3). For example, if $\partial / \partial \psi$ and $\partial / \partial z$ are standard generators of the (local) $\mathbb{R} \times \mathbb{R}$ action, then linear combinations such as $K=\partial / \partial \psi+\partial / \partial z$ are non-hypersurface orthogonal Killing fields.

Nevertheless, all such solutions do admit, of course, hypersurface orthogonal Killing fields and so may be written in the form (2.1). For the remainder of the paper, we thus assume that a Weyl solution has the form (2.1) locally. In addition, we will always work with the $\mathbb{Z}$-quotient of the metric (2.1) and so consider Weyl solutions as warped products of the form $V \times{ }_{f} S^{1}$; it will not be assumed in general that $V$ is simply connected.

Duality. Observe that Weyl solutions naturally come in 'dual' pairs. Namely the Ricci-flat 4-manifold $\left(N, g_{N}\right)$ has the form

$$
g_{N}=g_{V}+f^{2} d \phi^{2}+u^{2} d t^{2},
$$

and so both $M_{u}=V \times_{f} S^{1}$ and $M_{f}=V \times_{u} S^{1}$ are static vacuum solutions on the 3-manifolds, with potentials $u$, resp. $f$. Consider the product of the lengths of circles, or equivalently, the area of the torus fiber in $N$,

$$
r=f \cdot u \text {. }
$$

This is a globally defined positive harmonic function on $\left(V, g_{V}\right)$. To see this, on $M=M_{u}$, by (2.5), we have

$$
0=\Delta u=\Delta_{V} u+<\nabla \log f, \nabla u>,
$$

and the same formula, with $u$ and $f$ reversed, holds on $M_{f}$. Hence

$$
\Delta_{V} f u=f \Delta_{V} u+u \Delta_{V} f+2<\nabla u, \nabla f>=0 .
$$

Charts. We now describe a collection of preferred charts in which to express the Weyl solution $(M, g)$; this description is due to Weyl [Wl]. The surface $V$ may be partitioned into a collection of maximal domains $V_{i}$ on which the harmonic conjugate $z$ of $r$ is single-valued, so that $F=r+i z$ is a well-defined holomorphic function from $V_{i}$ into the right half-plane $\mathbb{C}^{+}=\{(r, z): r>0, z \in \mathbb{R}\}$. One might also pass to a suitable cover, for instance the universal cover, of $V$ to obtain a globally defined conjugate harmonic function, but it is preferable not to do so. 
Now each $V_{i}$ may be further partitioned into a collection of domains $V_{i j}$ on which $F$ is a conformal embedding into $\mathbb{C}^{+}$, so that $\left.g\right|_{V_{i j}}=F^{*}\left(d r^{2}+d z^{2}\right)$. We will thus simply view $V_{i j}$ as a domain in $\mathbb{C}^{+}$, with $g_{V}$ a metric pointwise conformal to the flat metric $d r^{2}+d z^{2}$.

It follows that the corresponding domain $M_{i j}=V_{i j} \times_{f} S^{1}$ is embedded as a domain $\Omega=\Omega_{i j}$ in $\mathbb{R}^{3}$ endowed with cylindrical coordinates $(r, z, \phi), \phi \in[0,2 \pi)$ with the background (unphysical) complete flat metric $d r^{2}+d z^{2}+r^{2} d \phi^{2}$. We note that all the data above are canonically determined by the two Killing fields on $N$ and thus the coordinates $(r, z, \phi)$ are called canonical cylindrical or Weyl coordinates for $(M, g)$. Of course $\Omega$ is axially symmetric, i.e. symmetric w.r.t. rotation about the $z$-axis.

To express the metric $\left.g\right|_{\Omega}$ in these coordinates, the field equations (0.1) imply that the function

$$
\nu=\log u
$$

is an axially symmetric (independent of $\phi$ ) harmonic function on $\Omega \subset \mathbb{R}^{3}$; this again follows in a straightforward way from computation of the Laplacian of $u$ and $f$ on $M_{f}$ and $M_{u}$ as above. A computation of the conformal factor for the metric $g_{V}$, c.f. [Wd,Ch.7.1], then leads to the expression of $g$ in these coordinates:

$$
g=u^{-2}\left(e^{2 \lambda}\left(d r^{2}+d z^{2}\right)+r^{2} d \phi^{2}\right)
$$

where $\lambda$ is determined by $\nu$ as a solution to the integrability equations

$$
\lambda_{r}=r\left(\nu_{r}^{2}-\nu_{z}^{2}\right), \lambda_{z}=2 r \nu_{r} \nu_{z} .
$$

The equations (2.13) mean that the 1-form $\omega=r\left(\nu_{r}^{2}-\nu_{z}^{2}\right) d r+2 r \nu_{r} \nu_{z} d z$ is closed on $\Omega$.

Conversely, given any axially symmetric harmonic function $\nu$ on a connected open set $\Omega$ in $\mathbb{R}^{3}$, if the closed 1-form $\omega$ is exact, (for example if $\pi_{1}\left(\Omega \cap \mathbb{C}^{+}\right)=0$ ), the equations (2.13) determine $\lambda$ up to a constant and the metric (2.12) gives a solution to the static vacuum equations with $S^{1}$ symmetry. (The addition of constants to $\nu$ or $\lambda$ changes the metric at most by diffeomorphism or homothety).

It is remarkable that solutions to the non-linear vacuum equations (0.1) can be generated in this way by solutions to the linear Laplace equation on $\mathbb{R}^{3}$.

Remark 2.3. (i). The Levi-Civita solutions in $\S 1$ are all Weyl solutions. However, the expressions for the A1-A3 metrics in (0.4),(1.7),(1.8) and the B1 metric in (0.9) are not in Weyl canonical coordinates. Note that the quotients of the A2 metric discussed above are no longer Weyl solutions, although they could be considered as local Weyl solutions; the $\mathbb{R}$-action is only locally defined on the quotients.

(ii). There seem to be no known Weyl solutions which can not be expressed globally in the form (2.12).

Fixed Point Set. The behavior of solutions at the part of $\partial M$ where either one of the two $S^{1}$ or $\mathbb{R}$ actions on $N$ has fixed points requires special considerations. This is of course the locus where $u=0$ or $f=0$, and hence includes the part $\bar{\Omega} \cap A$ of the $z$-axis $A=\{r=0\}$ in any canonical coordinate chart. It is not necessarily the case however that this locus is contained in $A$, c.f. the end of Example 2.10.

Note that given any Weyl solution (2.12), any covering of $\mathbb{R}^{3} \backslash A$ induces another solution of the form (2.12), but with $\phi$ parametrizing a circle of length $2 \pi k$. For the universal cover $(k=\infty)$, the $\phi$-circle is replaced by a line. In fact, (2.12) is well-defined when $\phi$ runs over any parameter interval $[0,2 \pi \alpha)$. Observe however that any asymptotically flat Weyl solution must have $\alpha=1$, since the metric must be smooth near infinity. Thus, we will assume $\alpha=1$ in the following, unless stated otherwise. 
Now suppose there is an open interval $J$ in $A$ such that the functions $u$ and $\lambda$, and hence the form (2.12) extend continuously to $J$. The form $g$ then represents a continuous metric in a neighborhood of $J$ if and only if the elementary flatness condition

$$
\lambda=0
$$

is satisfied on $J$. On intervals where (2.14) does not hold, the metric $g$ has cone singularities, so that it is not locally Euclidean. From (2.13), it is clear that if $\lambda$ has a $C^{1}$ extension to $J$, then $\lambda$ is constant on $J$. However, such constants may vary over differing components of $\bar{\Omega} \cap A$. This will be analysed further in Remark 2.8 .

For the remainder of $\S 2$, we assume that

$$
M=\Omega,
$$

so that the Weyl solution is given globally in the form (2.12). Let $I$ be the set where $\nu=-\infty$, i.e. the $G_{\delta}$ set in $\Omega \subset \mathbb{R}^{3}$ given by

$$
I=\bigcap_{n} \nu^{-1}(-\infty, n)
$$

where $n$ runs over negative integers. It is usually assumed in physics that $I$ is non-empty, although this need not be the case; this will also be discussed further below. The set $I$ corresponds to the horizon $\Sigma$ of the Weyl solution $(M, g)$, since $u=0$ on $I$. This correspondence is formal however, since the geometry and topology of $I \subset \mathbb{R}^{3}$ is very different than that of $\Sigma \subset(\bar{M}, g)$, c.f. most of the examples below. For the same reasons, although $M=\Omega$ topologically, the metric boundary $\partial M$ of $(M, g)$ is (most always) very different than the Euclidean boundary of $\Omega \subset \mathbb{R}^{3}$.

In the following, we discuss some of the most significant possible behaviors for the potential function $u$, and the associated Weyl solution, in order to illustrate the breadth of these solutions. The discussion is by no means complete or exhaustive.

\section{(I). $\bar{\Omega}$ compact.}

Let $M=\Omega$ be any bounded, $C^{\infty}$ smooth axisymmetric domain (i.e. connected open set with smooth compact boundary) in $\mathbb{R}^{3}$ and let $\phi$ be any $C^{k, \alpha}$ function on $\partial \Omega, k \geq 0, \alpha \in(0,1)$, which is axially symmetric about the $z$-axis. For simplicity, assume that $\Omega \cap \mathbb{C}^{+}$is simply connected. Let $\nu$ be the solution to the Dirichlet problem

$$
\Delta \nu=0,\left.\nu\right|_{\partial \Omega}=\phi .
$$

Then $\nu$ is also axi-symmetric about the $z$-axis, and hence $\nu$ generates a Weyl solution as in (2.12).

Suppose that for a given $k \geq 1 \alpha \in(0,1), \phi$ as above is $C^{k, \alpha}$ on $\partial \Omega$, but is nowhere $C^{k+1}$ on $\partial \Omega$. Then $\nu$ extends to a $C^{k, \alpha}$ function on the Euclidean closure $\bar{\Omega}$ and hence, from (2.13), the function $\lambda$ in (2.12) is also uniformly bounded. This means that the metric $g$ is quasi-isometric to the flat metric on $\Omega$, and hence the metric boundary of $\Omega$ w.r.t. the Weyl metric $g$ is the same as its Euclidean boundary. Since $\nu$ is not $C^{k+1}$ anywhere on $\partial \Omega$, this solution $M=\Omega$ is maximal, i.e. admits no larger static vacuum extension; $C^{2}$ smooth solutions of the static vacuum equations are analytic. Further $\nu$ is bounded, so that $u=e^{\nu}$ is bounded away from 0 , and hence the solution $(\Omega, g)$ has no horizon.

As noted in $\S 0$, the presence of the boundary $\partial M$ is physically assumed due to the presence of matter or field sources. Thus, at least when $k \geq 2$, the vacuum solution $(M, g)$ can be extended to a larger space-like domain $\left(M^{\prime}, g^{\prime}\right) \supset(M, g)$ with non-zero stress-energy $T$ in $M^{\prime} \backslash M$.

On the other hand, if $k=0$ above, then the geometry of the metric boundary $(\partial M, g)$ will in general be very different than the smooth geometry of $\partial \Omega$ in $\mathbb{R}^{3}$. Further, one can of course consider non-smooth domains $\Omega \subset \mathbb{R}^{3}$ in this situation. These remarks indicate that the structure of the metric boundary $\partial M$ seemingly can be quite arbitrary. 
For the remainder of this section, we assume that $\bar{\Omega}$ is non-compact in $\mathbb{R}^{3}$. The same remarks as above hold for non-compact domains with smooth (surface) boundary. Thus for example $M=\Omega$ might have infinitely many ends if $\partial \Omega$ is non-compact, showing that the assumption (i) in Theorem 0.3 is necessary. For simplicity, we only consider the following situation from now on.

(II). Suppose

$$
\operatorname{dim}_{\mathcal{H}} \partial \Omega \leq 1,
$$

where the boundary is in the topology of $\mathbb{R}^{3}$ and $\operatorname{dim}_{\mathcal{H}}$ is the Hausdorff dimension. Thus $\partial \Omega$ is a closed set of capacity 0, c.f. [H, Thm.5.14], and so in particular is a polar set. Clearly $\bar{\Omega}=\mathbb{R}^{3}$.

(A).(Positive Case). Suppose that $\nu$ is locally bounded above, i.e.

$$
\sup _{B_{x}(1)} \nu<\infty, \forall x \in \partial \Omega \text {. }
$$

It follows, c.f. [H, Thm.5.18] that $\nu$ extends uniquely to a globally defined subharmonic function on $\mathbb{R}^{3}$. Hence, one may use the value distribution theory of subharmonic functions on $\mathbb{R}^{3}$ to analyse the geometry of Weyl solutions.

The Riesz representation theorem c.f. [H,Thm.3.9], implies that any subharmonic function $\nu$ on $\mathbb{R}^{3}$ may be represented semi-globally, i.e. on $B(R)=B_{0}(R) \subset \mathbb{R}^{3}$ for any $R<\infty$, as

$$
\nu(x)=-\int_{B(R)} \frac{1}{|x-\xi|} d \mu_{\xi}+h(x),
$$

where $d \mu_{\xi}$ is a positive Radon measure on $B(R)$ called the Riesz measure of $\nu$ and $h$ is a harmonic function on $B(R)$; both $d \mu$ and $h$ are axi-symmetric if $\nu$ is. (A Radon measure is a Borel measure which is finite on compact subsets). For the moment, we only consider the situation where there exists $K<\infty$, independent of $R$, s.t.

$$
\int_{B(R)} \frac{1}{|x-\xi|} d \mu_{\xi} \leq K
$$

In this case, one obtains a global representation of $\nu$ as

$$
\nu(x)=-\int_{\mathbb{R}^{3}} \frac{1}{|x-\xi|} d \mu_{\xi}+h(x),
$$

where $d \mu_{\xi}$ is a positive measure and $h$ a harmonic function on $\mathbb{R}^{3}$. (In (D) below, we briefly discuss the situation where (2.19) is not assumed). In particular, if $\nu$ is uniformly bounded above, say $\sup \nu=0$, then the Liouville theorem for harmonic functions implies that $h \equiv 0$, and one has the expression

$$
\nu(x)=-\int_{\mathbb{R}^{3}} \frac{1}{|x-\xi|} d \mu_{\xi}
$$

Note that since $\nu$ is harmonic on $\Omega$,

$$
\bar{I} \subset \operatorname{suppd} \mu \subseteq \partial \Omega,
$$

but in many situations, as will be seen below, the first inclusion is strict.

\section{(A)(i). Pure harmonic potentials.}

Suppose that $\nu$ is a smooth harmonic function defined on all of $\mathbb{R}^{3}$, so that $\nu=h$ in (2.20). It is clear that in this case $\Sigma=\emptyset$ in the sense that $I=\emptyset$ in $\mathbb{R}^{3}$. Since $\nu$ is axisymmetric, $\nu$ may be viewed as an expansion in Legendre polynomials, i.e.

$$
\nu=\sum_{k \geq 0} a_{k} R^{k} \cdot P_{k}\left(\frac{z}{R}\right),
$$

where $R^{2}=r^{2}+z^{2}$. For instance, $R \cdot P_{1}\left(\frac{z}{R}\right)=z, R^{2} \cdot P_{2}\left(\frac{z}{R}\right)=3 z^{2}-R^{2}$. 
While these solutions are defined on all of $\mathbb{R}^{3}$, no such solution gives a complete Weyl metric $g$ on $M=\mathbb{R}^{3}$, by Theorem 1.1. For instance, for $\nu=z$, the Weyl metric is

$$
\left.g=e^{-2 z}\left(e^{-r^{2}}\left(d r^{2}+d z^{2}\right)+r^{2} d \phi^{2}\right)\right), u=e^{z} .
$$

Any straight ray in the $(r, z)$ half-plane has finite length in this metric, except a ray parallel to the negative $z$-axis. The horizon $\Sigma$ occurs formally at $\{z=-\infty\}$, of infinite $g$-distance to any point in $\mathbb{R}^{3}$.

\section{(A)(ii). Newtonian potentials.}

Suppose that $h=0$ in (2.20), so that $\nu$ is the Newtonian potential of an axisymmetric positive mass distribution $d \mu$ as in (2.21). This situation corresponds exactly to the Newtonian theory of gravity, (or equivalently the electrostatics of a positively charged distribution). While there is a vast classical literature on this subject, we will only consider the most interesting situation where

$$
\operatorname{suppd} \mu=\bar{I},
$$

so that $\nu$ approaches $-\infty$ on a dense set in supp $d \mu$.

The following Lemma characterizes this situation.

Lemma 2.4. Let $d \mu$ be an axi-symmetric positive Radon measure on $\mathbb{R}^{3}$. Then

$$
\operatorname{suppd} \mu=\bar{I} \Leftrightarrow \operatorname{suppd} \mu \subset A \text {. }
$$

Proof: Suppose first that supp $d \mu$ is not contained in $A$. Since $d \mu$ is axially symmetric, part of supp $d \mu$, namely the part not contained in $A$, is then given by a union of circles about the $z$-axis. Suppose first that there is a circle $C$ which is an isolated component of supp $d \mu$, so that $\left.d \mu\right|_{C}$ is a multiple of Lebesgue measure on $C$. This case has been examined in [Wl], [BW], and we refer there for details. In particular in this case the potential $\nu$ is bounded below on and near $C$, and hence supp $d \mu \neq \bar{I}$. If $C$ is not isolated, then using (2.21), the same reasoning holds, since the measure $d \mu$ is then even less concentrated on the circles.

On the other hand, if supp $d \mu \subset A$, then $d \mu$ is a positive Radon measure on $A$. Standard measure theory implies that the upper density of $d \mu$ w.r.t. Lebesgue measure $d A$ at $a \in A$, i.e. $\limsup _{r \rightarrow 0} \frac{\mu\left(B_{a}(r)\right)}{r}$, is positive, for Lebesgue almost all $a \in \operatorname{suppd} \mu$. From the expression (2.21), it is clear that for any such $a, \nu(x) \rightarrow-\infty$ as $x \rightarrow a$. This gives the converse.

For the remainder of the discussion in (A), we assume (2.23) holds. From the theory of subharmonic functions on $\mathbb{R}^{3}$, the set $I$ given by (2.15) may be an arbitrary $G_{\delta}$ set in $A \subset \mathbb{R}^{3}$, i.e. a polar set in $A$. Since countable unions of polar sets are polar, note that $I$ is not necessarily closed in $A \subset \mathbb{R}^{3}$. (For example, let $\left\{z_{i}\right\} \in A$ be an increasing sequence converging to a limit point $z$, with say $\operatorname{dist}\left(z_{i}, z_{i+1}\right)=i^{-2}$, and let $d \mu=\sum 2^{-i} \delta_{z_{i}}$, where $\delta_{z_{i}}$ is the Dirac measure based at $z_{i}$. Then $I=\left\{z_{i}\right\}$ and $\left.\operatorname{supp} d \mu=\left\{z_{i} \cup z\right\}\right)$.

Given any $x \in \mathbb{R}^{3}$, let $m_{x}(r)$ be the mass of the measure $d \mu$ in the ball $B_{x}(r)$, i.e.

$$
m_{x}(r)=\int_{B_{x}(r)} d \mu
$$

This is a non-negative increasing function on $\mathbb{R}^{+}$, for any given $x$ and the limit

$$
m=\lim _{r \rightarrow \infty} m_{x}(r)>0,
$$

is the total mass of $d \mu$. This agrees, up to a universal constant factor, with the (ADM or Komar) mass in general relativity, when the latter is defined, and with (0.7) for solutions with pseudocompact boundary. Note that one may have $m=+\infty$.

Lemma 2.4 and a standard result from potential theory, c.f. [H, Thm.3.20], characterize the possible Riesz measures satisfying (2.23). 
Lemma 2.5. A necessary and sufficient condition that a positive Radon measure $d \mu$ is the Riesz measure of an axi-symmetric subharmonic function $\nu$ on $\mathbb{R}^{3}$ with sup $\nu=0$ and supp $d \mu=\bar{I}$ is that supp $d \mu \subset A$, and, for any given $x \in A$,

$$
\int_{1}^{\infty} \frac{m_{x}(r)}{r^{2}} d r<\infty
$$

It is easy to see that a Weyl solution $(M, g)$ generated by a potential $\nu$ as in $(2.21)$ for which $\operatorname{supp}$ $d \mu=\bar{I}$ is a compact subset of the axis $A$, is asymptotically flat, in the sense preceding Theorem 0.1 . Further, the simplest or most natural surfaces enclosing any finite number of compact components of $\bar{I}$, and intersecting $A$ outside $\bar{I}$, are 2-spheres in $M$. Of course if suppd $\mu \subset A$ is non-compact, then the solution cannot be asymptotically flat. A simple example is the solution generated by the measure

$$
d \mu=\frac{1}{1+|\zeta|} d A_{\zeta}
$$

where $\zeta$ parametrizes $A$ and $d A$ is Lebesgue measure on $A$. Observe also that such solutions do not have pseudo-compact boundary.

It is worthwhile to discuss some standard examples of Weyl solutions and their corresponding measures.

Example 2.6. (i).(Curzon Solution). From the point of view of the Riesz measure, perhaps the simplest example is the measure $d \mu$ given by a multiple of the Dirac measure at some point on $A$, so that $\nu=-m / R, R(x)=|x|$, is a multiple of the Green's function on $\mathbb{R}^{3}$. This gives rise to the Curzon (or monopole) solution, c.f. [Kr, (18.4)],

$$
g_{C}=e^{2 m / R}\left[e^{-m^{2} r^{2} / R^{4}}\left(d r^{2}+d z^{2}\right)+r^{2} d \phi^{2}\right],
$$

with $u=e^{-m / R}$. Here $\Omega=\mathbb{R}^{3} \backslash\{0\}, \partial \Omega=\{0\}$, and it is often stated that $g_{C}$ has a point-like singularity (monopole) at the origin. However the geometry of $\partial M$ is very different than that of a point. Namely the circles about the $z$-axis have length diverging to infinity as $R \rightarrow 0$. Thus, small spheres $R=\epsilon$ about $\{0\}$ become very long in the $\phi$ direction, and very short in the transverse $\theta$ direction, forming a very long, thin cigar. In particular, as a metric space, $\partial M=\mathbb{R}$. This is the first example where $\partial M$ is non-compact but pseudo-compact. Of course $\partial M=\Sigma$, so that $(M, g)$ is complete away from $\Sigma$. A more detailed analysis of the Curzon singularity is given in [SS].

Note that one could not have solutions with both directions expanding at $\partial M$, so that area $\partial M=\infty$, with $\partial M$ pseudo-compact. This can be seen by use of minimal surface arguments, c.f. [G].

(ii). (Schwarzschild Solution). The Schwarzschild metric (0.4) is a Weyl metric, with measure $d \mu=\frac{1}{2} d A$ on $[-m, m]$, where $d A$ is the standard Lebesgue measure on $A$. The resulting potential $\nu$ in $(2.21)$ is the Newtonian potential of a rod on the z-axis with mass density $\frac{1}{2}$, given by

$$
\nu_{S}=\frac{1}{2} \log \left(\frac{R_{+}+R_{-}-2 m}{R_{+}+R_{-}+2 m}\right), \text { where } R_{ \pm}^{2}=r^{2}+(z \pm m)^{2} .
$$

As mentioned before, the horizon $\Sigma$ here is a smooth totally geodesic 2 -sphere of radius $2 m$ and $\partial M=\Sigma$.

The Weyl solution generated by the potential $a \cdot \nu_{S}$, for $\nu_{S}$ as in (2.29) with $a>0$ and $a \neq 1$, is not isometric or homothetic to the Schwarzschild metric. The associated Weyl metric is no longer smooth up to the horizon; in fact $\Sigma$ is not even a 2-sphere unless $a=1$. 
Remark 2.7. More generally, consider any Weyl solution generated by a Riesz measure $d \mu$ satisfying (2.23). Observe that $f=\frac{r}{u}$, the length of the $\phi$ circles in the Weyl metric, stays bounded away from 0 and $\infty$ on approach to supp $d \mu$, if and only if

$$
\log r-C \leq \nu \leq \log r+C,
$$

for some $C<\infty$, since $\nu=\log u$. From the expression (2.21), this occurs only for the Schwarzschild potential $\nu_{S}$. Briefly, the reason for this is as follows. The estimate (2.30) implies that the potential $v=\nu-\nu_{S}$ is bounded and given by convolution of $d i s t^{-1}$ with a signed Radon measure $d \lambda$. However, as in the proof of Lemma 2.4, if $v$ is bounded then one sees that necessarily $d \lambda<<d A$ and further the Radon-Nikodym derivative $d \lambda / d A$ is 0 , a.e. $(d A)$. In other words, any point of non-zero density for $d \lambda$ w.r.t. $d A$ gives rise to approximating points on which $\nu$ is unbounded. It follows that $d \lambda=$ 0 , and hence $v=0$.

In all other cases, where $f \rightarrow 0$ on approach to a region in supp $d \mu$, the length of the $\phi$ circles goes to 0 , and hence this portion of $\Sigma$ is singular, of dimension $\leq 1$, while where $f \rightarrow \infty$, the length of the circles goes to $\infty$ and the corresponding portion of $\Sigma$ is singular and non-compact, (as in the Curzon solution). Note that if supp $d \mu$ is compact, then in all cases, $\partial M$ is pseudo-compact.

Thus among the Weyl solutions given by a Newtonian potential, only the Schwarzschild metric is smooth up to $\Sigma$. This gives a very simple (local) confirmation of Theorem 0.1 in this special case.

Example 2.6.(iii). (Superposition/Multiple Holes). Subharmonic functions of the form (2.21) form a convex cone. In particular, one thus has a natural linear superposition principle for Weyl solutions. This feature is another remarkable property of Weyl solutions.

For example, one may choose the measure $d \mu=\frac{1}{2} d A$ on two, or any number of disjoint intervals $\left\{I_{j}\right\}$ on the axis $A$, provided (2.27) holds. These correspond to solutions with 'multiple black holes', each interval $I_{j}$ giving a component of $\Sigma$ which is a 2 -sphere of radius equal to the length of $I_{j}$. Although such solutions are essentially smooth up to $\Sigma$, they do not define smooth vacuum solutions on $\mathbb{R}^{3} \backslash \cup B_{j}$. There are cone singularities, (called struts or rods in the physics literature), along geodesics (corresponding to $A \backslash\left\{I_{j}\right\}$ ) joining the 2-spheres of $\Sigma$, so that the metric $g$ is not locally Euclidean along such curves. Thus, the elementary flatness condition (2.14) is violated on $A \backslash \cup I_{j}$. Nevertheless, the curvature of such metrics is uniformly bounded everywhere. These cone singularities must be considered part of $\partial M$, so $\partial M$ in this case is a union of 2 -spheres joined by a collection of curves and thus connected.

Of course, the black hole uniqueness theorem, Theorem 0.1, also implies that such solutions cannot be smooth everywhere, when the number of intervals is finite. However, the proof of this result strongly uses the asymptotically flat assumption. It seems to be unknown whether there are any Schwarzschild type metrics with infinitely many black holes, i.e. metrics complete away from $\Sigma$ and smooth up to $\Sigma$ with $\Sigma$ consisting of infinitely many 2-spheres, and which satisfy (2.17) or (2.21). It is natural to conjecture that such solutions do not exist, c.f. however the end of Remark 2.8 below.

Remark 2.8. Whenever supp $d \mu \subset A$ is not connected but compact, there will exist such cone singularities on $A \backslash \operatorname{supp} d \mu$. When supp $d \mu$, or a sufficiently small smoothing of supp $d \mu$, is interpreted to represent a matter source, then this statement corresponds exactly to the fact that there are no equilibrium (i.e. time-independent) many-body solutions in Newtonian gravity of this character, c.f. $[\mathrm{Bn}]$. Even when supp $d \mu$ is non-compact and disconnected, this seems very likely to be true, c.f. the expressions for sums of Schwarzschild rods (2.29) in [IK, p.336-337], which generalize to infinitely many rods.

The components $\left\{C_{i}\right\}$ of $A \backslash \operatorname{supp} d \mu$ represent idealized matter sources (struts or rods) keeping the components of supp $d \mu$ apart in static equilibrium. The cone angle $\alpha=\alpha_{i}$ is constant on each 
$C=C_{i}$, and corresponds to a concentration of scalar curvature on $C \subset \bar{M}$ given by a multiple of the Lebesgue measure on $C$

$$
s=(1-\alpha) d A_{C},
$$

Thus the vacuum equations (0.1) are not satisfied across $\left\{C_{i}\right\}$. If a very small tubular neighborhood of radius $r$ of such a rod is smoothly filled in with a perfect fluid source of constant pressure $\rho$ and energy density $\mu$, then one has the relation $\lim _{r \rightarrow 0} r \rho=-\lim _{r \rightarrow 0} r \mu>0$. The effective mass of such rods is zero, i.e. they do not contribute to the gravitational potential $\nu$, c.f. [I2] for a detailed discussion.

By passing to covering spaces, it is always possible to create such cone singularities in Weyl solutions, even if none existed to begin with. For instance, for the Schwarzschild solution (0.4), with potential (2.29), take any covering, including the universal covering, of $\mathbb{R}^{3} \backslash A=\left(S^{2} \backslash\{a \cup-a\}\right) \times \mathbb{R}^{+}$, where $\{a,-a\}$ are two antipodal points on $S^{2}$. This gives a solution whose metric completion has cone singularities along two (radial) geodesics starting at the antipodal points on $S^{2}=\Sigma$ and going to infinity.

Note that this discussion assumes that $\nu$ is given by a Newtonian potential (2.21). In fact, there are Weyl solutions $(M, g, u)$ everywhere smooth up to the axis $A$, with $\Sigma=\partial M$ disconnected, with no cone singularities or struts keeping the components of $\Sigma$ apart. Namely, the B1 solution (0.9), dual to the Schwarzschild solution, has this property.

Another, more remarkable, example is given in [KN]. These authors construct a Weyl solution of the form (2.12), which is complete away from $\Sigma$ and smooth up to $\Sigma$, with $\Sigma$ consisting of infinitely many Schwarzschild-like 2 -spheres. In fact, the solution is periodic in the $z$-direction. This metric is not of the form (2.21), but is a limit of a sequence of solutions of the form (2.20), c.f. (D) below.

Remark 2.9. If $(M, g, u)$ is a Weyl solution of the form (2.12) with $\nu=\nu_{u}=\log u$, then the dual solution $\left(M^{\prime}, g^{\prime}, f\right)$, discussed in (2.9), is also a Weyl solution of the form (2.12), with potential $\nu_{f}$ $=\log f$ given by

$$
\nu_{f}=\log r-\nu_{u}
$$

Hence if one potential is Newtonian, the dual one is not. Note that the sets $I_{u}, I_{f}$ where $\nu_{u}$ and $\nu_{f}$ are $-\infty$ are disjoint, with $\bar{I}_{u} \cup \bar{I}_{f}=A$. Hence, if $\nu_{u}$ is a Newtonian potential with supp $d \mu$ compact, so that the associated Weyl solution is asymptotically flat, then the dual Weyl solution is asymptotically cylindrical.

Another example of a potential where both terms in (2.20) are non-trivial is the situation considered (locally) in [GH], where $h$ is a smooth axi-symmetric harmonic function defined on a neighborhood of supp $d \mu$, c.f. Remark 1.5. As vacuum solutions, these metrics cannot be complete away from $\Sigma$, as in the discussion on pure harmonic potentials.

This completes our discussion of the Positive Case.

(B).(Negative Case). Under the assumption (2.16), suppose now instead that $\nu$ is locally bounded below in $\mathbb{R}^{3}$, i.e.

$$
\inf _{B_{x}(1)} \nu>-\infty, \forall x \in \partial \Omega .
$$

Then $\nu$ extends uniquely to a globally defined superharmonic function on $\mathbb{R}^{3}$. Exactly the same discussion as in (A) above holds here, under the substitution $\nu \rightarrow-\nu$. (This corresponds to the transformation $u \rightarrow u^{-1}$ following (1.5)). In this case, the Riesz measure is a negative measure, so that one has solutions with negative mass. Note that here the potentials $\nu$ or $u$ are unbounded above within supp $d \mu$, i.e. $u$ or $\nu$ go to $+\infty$.

(C).(Mixed Case). Next, one may superimpose Weyl solutions with positive and negative measures $d \mu$, i.e. consider $\nu$ of the form (2.21), with $d \mu$ a signed Radon measure. For example, 
one may form dipole-type solutions with potential of the form $\nu=\nu_{+}+\nu_{-}$where $\nu_{+}$and $\nu_{-}$are (for instance) Curzon or Schwarzschild solutions of positive and negative mass placed at different regions on the axis. This gives for instance examples of asymptotically flat solutions with mass $m$ assuming any value in $\mathbb{R}$.

More generally, since positivity is no longer assumed, the measure $d \mu$ may be replaced by distributions, for example weak derivatives of measures.

Example 2.10. (Multipole Solutions). As a typical example, one may take potentials corresponding to derivatives of the Dirac measure based at a point $a \in A$, i.e. the multipole potentials,

$$
R^{-n-1} \cdot P_{n}\left(\frac{z}{R}\right)
$$

where $P_{n}$ is the $n^{\text {th }}$ Legendre polynomial, or arbitrary linear combinations of such; c.f. [MF, p.1276ff].

Such potentials are limits of combinations of Newtonian potentials with positive and negative mass. Thus, it is reasonable to expect that there are (Newtonian) equilibrium solutions, i.e. solutions with no cone singularities on the axis. This is proved in [Sz], where explicit equilibrium conditions are given. Note that one may have infinitely many multipole 'particles' in equilibrium.

Another example is the potential of a dipole ring

$$
\nu(x)=-\int_{C} \frac{z(x)}{|x-\xi|^{3}} d \xi,
$$

where $x=(r, z, \phi)$ and $d \xi$ is the Lebesgue measure on the unit circle $C=\{r=1\}$ in the $z=0$ plane. Here $\nu(x) \rightarrow-\infty$, as $x \rightarrow C$ along the rays $r=1, z>0$. Hence, in this case, the set $I=C$ is not contained in the axis $A$.

(D). (Limits). Finally, one may consider potentials which are limits of potentials of the type (A)-(C) above, (besides those in Remark 2.9, Example 2.10). We consider just one important instance of this here.

Example 2.11. (Kasner Metric). It is easily seen that the potential $\nu=\log r$ generates the flat metric

$$
g=d r^{2}+d z^{2}+d \phi^{2},
$$

on $\left(\mathbb{R}^{3}\right)^{+}$, with potential $u=r$. Observe that since the $\phi$-lines have constant length, the function $r$ is now an affine (in fact linear) function on $\left(\mathbb{R}^{3}\right)^{+}$.

On the other hand, an equally simple computation shows that the potential $\nu=a \cdot \log r$, for any $a \in \mathbb{R}$, generates the metric

$$
g=r^{2 a^{2}-2 a}\left(d r^{2}+d z^{2}\right)+r^{2-2 a} d \phi^{2}, u=r^{a} .
$$

Equivalently, setting $s=r^{a^{2}-a+1}$,

$$
g=d s^{2}+s^{\alpha} d z^{2}+s^{\beta} d \phi^{2}, u=s^{\gamma},
$$

where $\alpha=(2 a-2) /\left(a-1+a^{-1}\right), \beta=\left(2 a^{-1}-2\right) /\left(a-1+a^{-1}\right), \gamma=\left(a-1+a^{-1}\right)^{-1}$. Here $s \in \mathbb{R}^{+}, z \in \mathbb{R}$ and $\phi \in[0,2 \pi]$ or any other interval, including $\mathbb{R}$, (by passing to covering and quotient spaces). These metrics are all non-homothetic, provided $a \in[-1,0) \cup(0,1] ; a=0$ gives the flat metric with $u=1$ while $a=-1$ gives the $A 3$ metric (1.8).

The potential $\nu=a \cdot \log r$ can be considered as the limit

$$
\nu=\lim _{m \rightarrow \infty} a\left[\nu_{S}(m)-\log 2 m\right],
$$

where $\nu_{S}(m)$ is the Schwarzschild potential (2.29) of mass $m$. Thus it is a limit of potentials of the form (2.18), where both terms are non-zero, (the harmonic term $h$ is of course constant here). 
These metrics are dual, in the sense discussed in (2.9) to the Kasner (or Bianchi I) vacuum cosmological models, with homogeneous (flat) but anisotropic space-like hypersurfaces, c.f. [Wd, Ch.7.2]. It is easy to see that the Kasner metrics are the only Weyl solutions $(M, g)$ which have an isometric $\mathbb{R} \times \mathbb{R}$ action, even locally. (The axisymmetric potential $\nu$ on $\Omega \subset \mathbb{R}^{3}$ must be invariant under an orthogonal $\mathbb{R}$-action, hence giving a rotationally invariant harmonic function on $\mathbb{R}^{2}$. Thus the potential must be a multiple of $\log r$ ).

Consider these metrics on the quotient $M=\mathbb{R}^{+} \times S^{1} \times S^{1}$. In case $a>0$, we have $\alpha<0, \beta>0$, $\gamma>0$ and so $\Sigma=\partial M$. As in the discussion with the Curzon metric, the $z$-circles have unbounded length as $s \rightarrow 0$, so that $\partial M=\mathbb{R}$ and the levels $t=\epsilon,\left(t(x)=\operatorname{dist}_{g}(x, \partial M)\right)$, are long, thin cigars. Thus, $\partial M$ is non-compact, but pseudo-compact. The end of $\bar{M} \backslash U$ is obviously small.

If $a<0$, then $\alpha>0, \beta>0, \gamma<0$ and so $\Sigma=\emptyset$, (it occurs at infinity), with $\partial M=\{\mathrm{pt}\}$. In this case, the end of $\bar{M} \backslash U$ is not small; the area growth of geodesic spheres is $O\left(r^{1-\gamma}\right)$.

However, none of these solutions are asymptotically flat even in a weak sense, except of course when $a=1$. Namely, the curvature decays only quadratically in the $g$-distance to $\partial M$, i.e.

$$
|r|=O\left(t^{-2}\right),
$$

and not any faster. Hence, the case $a>0$ shows that the conclusion of Theorem 0.3 , (asymptotically flat or small ends), cannot be strengthened to only asymptotically flat ends, while the case $a<0$ shows that the assumption (ii) on $u$ in Theorem 0.3 is necessary.

Another metric of this (limit) type is that constructed in $[\mathrm{KN}]$, referred to in Example 2.8. This metric has the same asymptotics as the Kasner metric.

\section{Characterization of Asymptotically Flat Solutions.}

In this section, we prove Theorem 0.3. The proof of the first statement on finiteness of the number of ends is quite easy, so we begin with this.

Throughout this section, let $(M, g, u)$ be a static vacuum solution with $\partial M$ pseudo-compact. We recall from $\S 0$ that $M$ is connected and oriented. As in $\S 0$, let

$$
t(x)=\operatorname{dist}_{g}(x, \partial M),
$$

and suppose $U=t^{-1}\left(0, s_{o}\right)$, so that $\partial U \cap M$ is compact. For $r, s \geq s_{o}$, let $S(s)=t^{-1}(s), A(r, s)=$ $t^{-1}(r, s)$ be the geodesic spheres and annuli about $\partial U$. It is important to note that neither $S(s)$ nor $A(r, s)$ are necessarily connected, even if $M$ has only one end. (Of course if $E$ is a given end of $M$, then $S_{E}(s)=S(s) \cap E$ must be connected for some sequence $\left.s=s_{j} \rightarrow \infty\right)$. Let $S_{c}(s)$ and $A_{c}(r, s)$ denote any component of $S(s)$ resp. $A(r, s)$, so that $S(s)=\cup S_{c}(s), A(r, s)=\cup A_{c}(r, s)$. Of course $t$ is a proper exhaustion function on $\bar{M} \backslash U$, so that these sets have compact closure in $M$.

Let $\operatorname{diam}^{i} A_{c}(r, s)$ denote the intrinsic diameter of $A_{c}(r, s)$, i.e. the diameter of the connected metric space $\left(A_{c}(r, s), g\right)$.

Lemma 3.1. There exists a constant $d_{o}<\infty$, independent of $s$, such that the number of components of $A\left(\frac{1}{2} s, 2 s\right)$ is at most $d_{o}$ and

$$
\operatorname{diam}^{i} A_{c}\left(\frac{1}{2} s, 2 s\right) \leq d_{o} \cdot s
$$

In particular, the manifold $(\bar{M} \backslash U, g)$ has a finite number of ends $\left\{E_{i}\right\}$.

Proof: Consider first the 4-manifold $\left(N, g_{N}\right)$, as in $(0.2)$ which is smooth and Ricci-flat outside $\bar{U}=\pi^{-1}(U)$, where $\pi: N \rightarrow M$ is projection on the first factor. Since $\partial \bar{U} \cap N$ is compact, it follows from results of [Lu] that Lemma 3.1 holds on $N$, so that in particular $N$ has a finite number of ends. Since $N=M \times S^{1}, \bar{M} \backslash U$ also has a finite number of ends. 
The choice of the time parameter on $N$ defines a totally geodesic embedding $M \subset N$ and we have $\left.t_{N}\right|_{M}=t$ where $t_{N}(x)=\operatorname{dist}_{N}(x, \partial M), \partial M \subset-N$. A geodesic ball or annulus in $M$ embeds in the geodesic ball or annulus of the same size in $N$. Hence (3.1) also holds for $M$.

Lemma 3.1 of course proves the first statement of Theorem 0.3. Observe that the estimate (3.1) is invariant under rescaling of the metric $g$.

For the remainder of the proof, we (usually) work with a given end $E$ from the finite collection $\left\{E_{i}\right\}$. The main statement of Theorem 0.3 is that if

$$
\int^{\infty} \frac{1}{\operatorname{areaS_{E}(s)}} d s<\infty,
$$

then the end $E$ is asymptotically flat. The proof of this result is rather long, so we outline here the overall strategy. The asymptotic behavior of $(E, g, u)$ is studied in general by examining the structure of the possible tangent cones at infinity, defined below. Basically, tangent cones at infinity fall into two classes, according to whether the asymptotic geometry near a given divergent sequence of base points is non-collapsing or collapsing, c.f. Lemmas 1.3-1.4. The main point is to prove that under the bound (3.2), all tangent cones at infinity are flat manifolds, and further that no collapse behavior is possible. Once this is established, the proof that $(E, g, u)$ is asymptotically flat is relatively straightforward.

Apriori, the end $E$ may be very complicated topologically, for instance of infinite topological type; consider for instance that $E$ might be of the form $S_{\infty} \times S^{1}$, where $S_{\infty}$ is any non-compact surface of infinite topological type and one end. A main idea is to use the behavior of the potential function $u$, in particular its value distribution theory, to control the topology and geometry of $E$ in the large. We have already seen in $\S 2$ that the potential $u$ controls quite strongly the geometry of Weyl solutions. Lemma 3.6 below is the key technical lemma which expresses this control for general static vacuum solutions (with pseudo-compact boundary). Further remarks on the strategy of proof precede the Lemmas below.

The discussion to follow, until the end of Lemma 3.6, holds in general for ends $E$ of static vacuum solutions with compact boundary. The estimate (3.2) will only be used after this.

We now define the tangent cones at infinity of a given end $E$. (While this is a commonly used terminology, such limit metric spaces are not necessarily metric cones in general).

First, we recall by Theorem 1.1 that there is a constant $K<\infty$ such that, $\forall x \in M$,

$$
|r|(x) \leq \frac{K}{t^{2}(x)},|d \log u|(x) \leq \frac{K}{t(x)}
$$

The scale-invariant estimates (3.3) give quite strong initial control on the asymptotic geometry of $(E, g, u)$ which allows one to get started. Observe that an immediate consequence of $(3.1)$ and (3.3), by integration along paths in $A_{c}\left(\frac{1}{3} s, 3 s\right)$, is the following Harnack inequality:

$$
\frac{\text { supu }}{\text { infu }} \leq K_{1}
$$

where the sup and inf are taken over any component $A_{c}\left(\frac{1}{2} s, 2 s\right)$ and $K_{1}$ is independent of $A_{c}\left(\frac{1}{2} s, 2 s\right)$.

Let $x_{i}$ be any divergent sequence of points in $E$, with $t_{i}=t\left(x_{i}\right) \rightarrow \infty$. Consider the connected geodesic annuli $A_{i}=A_{i}(\kappa)=A_{c}\left(\kappa^{-1} t_{i}, \kappa t_{i}\right), x_{i} \in A_{i}$, w.r.t. the rescaled or blow-down metric

$$
g_{i}=t_{i}^{-2} \cdot g
$$

here $\kappa$ is any fixed positive constant $>1$. By the curvature estimate (3.3), the metrics $g_{i}$ have uniformly bounded curvature on $A_{i}$ - the curvature bound depends only on $K$ and $\kappa$. Further, by (3.1), the diameter of $A_{i}$ w.r.t. $g_{i}$ is also uniformly bounded. 
Hence, if the sequence is non-collapsing, i.e. if there is a lower volume bound $\operatorname{vol}_{g} A_{i} \geq \nu_{o} \cdot t_{i}^{3}$, for some $\nu_{o}>0$, (equivalent to $\operatorname{vol}_{g_{i}} A_{i} \geq \nu_{o}$ by scaling), then Lemma 1.3 implies that a subsequence of the pointed sequence $\left\{\left(A_{i}, g_{i}, x_{i}\right)\right\}$, converges smoothly, away from the boundary, to a limiting smooth metric $\left(A_{\infty}(\kappa), g_{\infty}, x_{\infty}\right)$. The limit is a solution of the static vacuum equations; as noted in Lemma 1.3, the potential $u$ is renormalized to $u_{i}=u / u\left(x_{i}\right)$, so that the limit potential $u_{\infty}$ satisfies $u_{\infty}\left(x_{\infty}\right)=1$, c.f. also (3.4). Choosing a sequence $\kappa_{j} \rightarrow \infty$ and a suitable diagonal subsequence, gives the maximal static vacuum solution $\left(A_{\infty}, g_{\infty}, u_{\infty}, x_{\infty}\right)$. Observe here also that the estimate (3.1) implies that $\partial A_{\infty}=\{\mathrm{pt}\}$.

On the other hand, if the sequence $\left(A_{i}, g_{i}\right)$ is collapsing, in the sense that $\operatorname{vol}_{g} A_{i}<<t_{i}^{3}$, as $t_{i} \rightarrow \infty$, (equivalent to $\operatorname{vol}_{g_{i}} A_{i} \rightarrow 0$ ), then Lemma 1.4 implies that $A_{i}$ is a Seifert fibered space or torus bundle over an interval. As discussed there, one may then pass to $\mathbb{Z}$ or $\mathbb{Z} \oplus \mathbb{Z}$ covers $\widetilde{A}_{i}(\kappa)$ of $A_{i}(\kappa)$ (or more precisely smooth interior approximations to $A_{i}(\kappa)$ ) to obtain a non-collapsing sequence $\left(\widetilde{A}_{i}(\kappa), g_{i}, x_{i}\right)$, smoothly convergent to a limit $\left(\widetilde{A}_{\infty}(\kappa), g_{\infty}, x_{\infty}\right) ;\left(g_{i}\right.$ here is lifted to the cover $\widetilde{A}_{i}$, as is $\left.x_{i}\right)$. As above, one may then choose a sequence $\kappa_{j} \rightarrow \infty$ and pass to a diagonal subsequence to obtain a maximal limit $\left(\widetilde{A}_{\infty}, g_{\infty}, u_{\infty}\right)$. This limit static vacuum solution has an isometric $\mathbb{R}$ action, or $\mathbb{R} \oplus \mathbb{R}$ action in the case of a rank 2 collapse. Hence by Proposition 2.2 it is a Weyl solution. In the latter case, the solution is then a (possibly flat) Kasner metric, c.f. Example 2.11. Topologically, the limit $\widetilde{A}_{\infty}$ is a trivial $\mathbb{R},($ or $\mathbb{R} \oplus \mathbb{R}$ ), bundle over a surface $V$, (or interval), again by Proposition 2.2 .

As in $\S 2$, we will always work in a $\mathbb{Z}$-quotient $\bar{A}_{\infty}$ of $\widetilde{A}_{\infty}$, (or $\mathbb{Z} \oplus \mathbb{Z}$ quotient in the case of rank 2 collapse), and finite covers $\bar{A}_{i}(\kappa)$ converging to $\bar{A}_{\infty}(\kappa)$. For any fixed $\kappa>0$, the manifolds $\left(\bar{A}_{i}(\kappa), g_{i}, x_{i}\right)$ thus have uniformly bounded curvature and diameter, a uniform lower bound on their volume, and converge smoothly to the limit $\left(\bar{A}_{\infty}(\kappa), g_{\infty}\right) \subset\left(\bar{A}_{\infty}, g_{\infty}\right)$. The limit has a free isometric $S^{1}$ or $S^{1} \times S^{1}$ action, and so in particular is an $S^{1}$ or $T^{2}$ bundle. Hence $\bar{A}_{i}(\kappa)$ is also topologically an $S^{1}$ or $T^{2}$ bundle, (although not metrically).

To be definite, the finite covers are chosen so that the length of the $S^{1}$ factor or factors at the base point $x_{i} \in \bar{A}_{i}(\kappa)$ converge to 1 in the limit.

Recall by Lemma 1.4 that the inclusion map of the fibers induces an injection on $\pi_{1}$. The coverings $\bar{A}_{i}(\kappa)$ are obtained by taking large finite unwrappings of the $S^{1}$ or $T^{2}$ fibers, (corresponding to taking subgroups of $\pi_{1}\left(S^{1}\right)$ or $\pi_{1}\left(T^{2}\right)$ of large but finite index). All finite covering spaces of $S^{1}$ or $T^{2}$ are still $S^{1}$ or $T^{2}$, and hence we may, and do, choose the unwrappings so that, as smooth manifolds,

$$
\bar{A}_{i}(\kappa)=A_{i}(\kappa)
$$

for any $\kappa>0$. In the limit, the unwrapping of the collapse thus just corresponds to expanding the length of the collapsing $S^{1}$ factor (or factors), preserving the holonomy, if any, of the $S^{1}$ bundle; compare with the proof of Proposition 2.2.

The limit spaces $\left(A_{\infty}, g_{\infty}, u_{\infty}, x_{\infty}\right)$ or $\left(\bar{A}_{\infty}, g_{\infty}, u_{\infty}, x_{\infty}\right)$ constructed above are called tangent cones at infinity of $(E, g, u)$. Note that such tangent cones are only attached to some subsequence of a given divergent sequence of base points $\left\{x_{i}\right\}$. Hence, apriori, the tangent cones at infinity could be highly non-unique as Riemannian manifolds. In general, there may be no relation between the geometry of different tangent cones based on (subsequences of) distinct divergent sequences $\left\{x_{i}\right\}$; for example, tangent cones based on sequences with $t\left(x_{i}\right)=2^{i^{2}}$ and $t\left(x_{i}\right)=2^{i^{3}}$. The tangent cones only detect behavior of the end $E$ in $g_{i}$-bounded distance to the base points $x_{i}$.

On the other hand, since tangent cones at infinity attached to any divergent sequence always exist, for any $s$ sufficiently large, say $s \geq s_{o}$, the geometry of $\left(A_{c}\left(\frac{1}{2}, 2\right), g_{s}\right)$ or $\left(\bar{A}_{c}\left(\frac{1}{2}, 2\right), g_{s}\right)$ is always close to that of some tangent cone at infinity $A_{\infty}$ or $\bar{A}_{\infty}$. Further, by construction, the tangent cones are always connected and, since $M$ is oriented, so is each tangent cone.

The following lemma is a typical application of the use of tangent cones at infinity. 
Lemma 3.2. Suppose the curvature $r$ decays faster than quadratically in the end $(E, g, u)$, i.e.

$$
|r|(x) \leq \frac{\varepsilon(t)}{t^{2}(x)}
$$

where $\varepsilon(t) \rightarrow 0$ as $t \rightarrow \infty$. Then there is a compact set $K \subset E$ such that $E \backslash K$ is diffeomorphic either to $\mathbb{R}^{3} \backslash B$ or to $\left(\mathbb{R}^{2} \backslash B\right) \times S^{1}$, where $B$ is a 3-ball, (resp. a 2-ball), i.e. E is of standard topological type. Further, the annuli $A_{E}\left(\frac{1}{2} s, 2 s\right)$ are connected, for all $s \geq s_{o}$, for some $s_{o}<\infty$.

Proof: By the preceding discussion, the condition (3.6) is equivalent to the statement that all tangent cones at infinity $\left(A_{\infty}, g_{\infty}\right)$ or $\left(\bar{A}_{\infty}, g_{\infty}\right)$ of $E$ are flat, (as well as connected and oriented). The two possible conclusions of Lemma 3.2 correspond to the two possibilities of non-collapse and collapse in the formation of the tangent cones.

Suppose first that $g$ is non-collapsing on $E$, i.e. there exists $\nu_{o}>0$ such that $\operatorname{vol}_{g} A_{c}\left(\frac{1}{2} s, 2 s\right) \geq$ $\nu_{o} \cdot s^{3}$, for all $s$ large, and all components $A_{c}$. Recall that $\partial A_{\infty}=\{p t\}$ in this situation. It then follows that for $s$ sufficiently large, each $A_{c}\left(\frac{1}{2} s, 2 s\right)$ is diffeomorphic, and almost isometric to the standard flat annulus $A=r^{-1}\left(\frac{1}{2} s, 2 s\right)$ in $\mathbb{R}^{3}, r(x)=|x|$, (away from the boundary). In fact, each tangent cone at infinity $A_{\infty}$ is isometric to $\mathbb{R}^{3} \backslash\{0\}$ in this situation. Here we are implicitly using the fact that the only complete oriented flat 3 -manifold with an isolated singularity is $\mathbb{R}^{3} \backslash\{0\}$, c.f. $[\mathrm{AC}]$ for example. Similarly, a smooth approximation to $S_{c}(s)$ is diffeomorphic and almost isometric to $S^{2}(s) \subset \mathbb{R}^{3}$.

By the isotopy extension theorem, these diffeomorphisms from $A_{c}\left(\frac{1}{2} s, 2 s\right)$ to the standard annulus may then be assembled to a global diffeomorphism, and almost isometry, of $E \backslash K$ into $\mathbb{R}^{3} \backslash B$, for some compact set $K \subset E$. We refer to [AC, Thm.1.18] for the proof of these statements, (in a slightly different but equivalent form), which are now quite standard. The main point is of course that since the family of annuli $A_{c}\left(\frac{1}{2} s, 2 s\right)$ as $s$ varies is topologically rigid, i.e. one has a unique topological type, there is no value of $s$ at which the topology can change or bifurcate.

Suppose on the other hand that $g$ is collapsing on $A_{c}\left(\frac{1}{2} s_{i}, 2 s_{i}\right)$, for some sequence $s_{i} \rightarrow \infty$, and some sequence of components $A_{c}$. As discussed above, one may then pass to suitable covers $\bar{A}_{i}=\bar{A}_{c}\left(\frac{1}{2} s_{i}, 2 s_{i}\right)$ so that, in a subsequence, $\left(\bar{A}_{i}, g_{i}\right)$ is diffeomorphic and almost isometric to its limit $\bar{A}_{\infty}\left(\frac{1}{2}, 2\right) \subset \bar{A}_{\infty}$. The maximal limit $\bar{A}_{\infty}$ is a flat manifold with either a free isometric $S^{1}$ or $S^{1} \times S^{1}$ action. Hence there are two possibilities for $\bar{A}_{\infty}$, namely either $V \times S^{1}$ or $\mathbb{R}^{+} \times S^{1} \times S^{1}$, where $V$ is a flat 2-manifold and the metric is a product metric on each $S^{1}$ factor. In the former case, the diameter estimate (3.1) implies, (as in the non-collapse case above), that $V$ is a complete flat cone, possibly with an isolated singularity at $\{0\}$. Hence, although these two possibilities for the limiting metric of $\bar{A}_{\infty}\left(\frac{1}{2}, 2\right)$ are distinct, both are the same topologically, i.e. $\bar{A}_{\infty}\left(\frac{1}{2}, 2\right)$ is topologically $I \times S^{1} \times S^{1}$.

Now recall from the discussion on tangent cones that $\bar{A}_{i}$ is diffeomorphic to $A_{c}\left(\frac{1}{2} s_{i}, 2 s_{i}\right)$; metrically $\bar{A}_{i}$ approximates one of the types of flat manifolds above, with $S^{1}$ factors shrinking to very short circles. In both cases, (a smoothing of) $\bar{S}_{c}\left(s_{i}\right)$ is diffeomorphic and almost isometric to a flat torus $T^{2}$.

In particular, the topological type of $\bar{A}_{i}$ is distinct from that of the annuli $A_{i}$ above in the noncollapse case, which are topologically always of the form $I \times S^{2} \subset \mathbb{R}^{3} \backslash\{0\}$, (for any choice of base point and component). This implies first that the family $\left\{A_{c}\left(\frac{1}{2} s, 2 s\right)\right\}$ must be collapsing for all $s$, as $s \rightarrow \infty$, and all components $A_{c}$. Second, the topological type of the annuli $\bar{A}_{s}$, and hence that of $A_{s}=A_{c}\left(\frac{1}{2} s, 2 s\right)$ is unique, and given for all $s$ large and all $c$ by $I \times S^{1} \times S^{1}$. Use of the isotopy extension theorem in the same way as above then proves that the end $E$ itself is diffeomorphic to $\left(\mathbb{R}^{2} \backslash B\right) \times S^{1}$. 
Remark 3.3. (i) It is easily seen from the vacuum equations (0.1) that the condition (3.6) is equivalent to

$$
|\nabla \log u|(x) \leq \frac{\varepsilon(t)}{t(x)}
$$

as $t \rightarrow \infty$, c.f. also the proof of Theorem 1.1 .

(ii). In the context of Lemma 3.2 , the tangent cones at infinity $\left(\bar{A}_{\infty}, g_{\infty}\right)$ may not be unique up to isometry in the collapse case, and so may vary within the moduli space $\mathcal{M}_{1}$ of flat product metrics of the form $V \times S^{1}$ or within the moduli space $\mathcal{M}_{2}$ of flat product metrics of the form $\mathbb{R}^{+} \times S^{1} \times S^{1}$.

Note that the moduli space $\mathcal{M}_{o}$ of flat metrics on $\mathbb{R}^{3}$ or $\mathbb{R}^{3} \backslash\{0\}$ is just one point, (c.f. again [AC] for the latter statement for example). Similarly, by the normalization preceding Lemma 3.2 that the $S^{1}$ factors have length 1 , the moduli space $\mathcal{M}_{2}^{\prime} \subset \mathcal{M}_{2}$ normalized in this way is also just one point. The moduli space $\mathcal{M}_{1}^{\prime} \subset \mathcal{M}_{1}$ where the $S^{1}$ factor has length 1 is naturally identified with $\mathbb{R}^{+}$, parametrized by the cone angle at $\{0\}$.

Observe however that these two moduli spaces $\mathcal{M}_{1}^{\prime}$ and $\mathcal{M}_{2}^{\prime}$ are disjoint; they cannot be connected (or even approximated) by a curve of flat metrics. Now the geometry of the annuli $\left(A_{E}\left(\frac{1}{2} s, 2 s\right), g_{s}\right)$ varies continously with $s$. By the remarks preceding Lemma 3.2, this induces a continuous variation of the possible tangent cones $\left(\bar{A}_{\infty}, g_{\infty}\right)$ in $\mathcal{M}_{1}^{\prime}$ or $\mathcal{M}_{2}^{\prime}$. Hence, on a given end $E$, one cannot obtain two different tangent cones, one of the form $V \times S^{1}$ and another of the form $\mathbb{R}^{+} \times S^{1} \times S^{1}$; c.f. also the proof of Lemma 3.7 below.

(iii). We will need a slight generalization of Lemma 3.2 for the next lemma below. Thus let $\gamma(s)$ be any properly embedded curve in $E$ with $t(\gamma(s)) \rightarrow \infty$ as $s \rightarrow \infty$, and suppose (3.6), (or (3.7)), holds in the balls $B_{\gamma(s)}(\delta \cdot t(\gamma(s)))$, for some fixed $\delta>0$. Then the conclusion of Lemma 3.2 also holds.

To see this, consider the blow-downs $g_{s}=t^{-2}(\gamma(s)) \cdot g$, based at $\gamma(s)$, and the associated tangent cones at infinity. The scale invariant condition (3.6), when applied to $B_{\gamma(s)}(\delta \cdot t(\gamma(s)))$, implies that all such tangent cones are flat in $\left(B_{x_{\infty}}(\delta), g_{\infty}\right)$, and thus flat everywhere in their maximal domain $A_{\infty}$, by the fact that smooth solutions of the vacuum equations are real analytic. The proof then proceeds exactly as in Lemma 3.2

To prove that the estimates (3.6) and (3.7) do in fact hold on $E$, we need to understand in more detail the value distribution of the potential $u$. The main result needed for this is given in Lemma 3.6, and then (3.6)-(3.7) follow rather easily in (3.17)-(3.18) below. However, some preliminary results are required for the proof of Lemma 3.6. The main difficulty is that $u$ may not, in this generality, be a proper function onto its image, (c.f. the remark following the proof of Lemma 3.4). Lemma 3.4 below is a slightly weaker substitute for this property.

Let $U=t^{-1}\left(0, s_{o}\right)$ be a neighborhood of $\partial M$ as in the beginning of $\S 3$. Let $\gamma(\tau)$ be a maximal flow line of $\nabla u$. We will say that $\gamma$ is divergent if $\gamma$ does not intersect $U$ at two different times, i.e. if $\gamma(\tau)$ exits $U$ at some time, then $\gamma(\tau)$ never reenters (a possibly distinct component of) $U$, and if further $\gamma(\tau)$ does not terminate at a critical set of $u$ in $\bar{M} \backslash U$ as $\tau \rightarrow \pm \infty$. It follows that if $\gamma$ is divergent, then $\gamma$ is complete in at least one direction, $(\tau \rightarrow+\infty, \tau \rightarrow-\infty$ or both $)$, and in any such direction, $\gamma(\tau)$ diverges to infinity in $M$. Since the potential $u$ has no local maxima or minima in $M$, the set of flow lines terminating on a critical set of $u$ in $M \backslash U$ is a closed set of measure 0 in $M$. This follows for instance from the fact that the measure $|d u| d A$, where $d A$ is Lebesgue measure on the level sets of $u$, is preserved under the gradient flow of $u$, and this measure tends to 0 on approach to critical points of $u$. Thus among the set of flow lines not joining points of $U$, the divergent flow lines are generic in terms of measure on $M$. Of course the flow lines are curves of steepest ascent for $u$ as $\tau$ increases, and of steepest descent for $u$ as $\tau$ decreases. 
Lemma 3.4. There exists a compact set $K \subset \bar{M}$, with $\bar{U} \subset K$, such that any divergent flow line $\gamma(\tau)$ intersects $K$.

Proof: Suppose that this were not the case, so that there exist, necessarily complete, flow lines $\gamma(\tau), \tau \in \mathbb{R}$, which do not intersect a given $K \supset \bar{U}$. We may choose $K$ sufficiently large so that $\gamma(\tau)$ is then contained in a fixed end $E \subset \bar{M} \backslash U$, since there are only finitely many ends.

Let $A_{\gamma}(\tau)=A_{\gamma}\left(\frac{1}{2} t(\gamma(\tau)), 2 t(\gamma(\tau))\right)$ be the component of the geodesic annulus containing the base point $\gamma(\tau)$ and let $E_{\gamma}$ be the part of $E$ swept out by such annuli, $E_{\gamma}=\cup_{\tau} A_{\gamma}(\tau) \subset E$. As $\tau \rightarrow \infty$, the function $u(\gamma(\tau))$ is monotone increasing.

If $u(\gamma(\tau))$ increases to $+\infty$, then $u \rightarrow \infty$ uniformly as $\tau \rightarrow \infty$ in $E_{\gamma}^{+}=\cup_{\tau>0} A_{\gamma}(\tau) \subset E$, by the Harnack estimate (3.4). Since $E$ is an end, there exists some sequence $\tau_{j} \rightarrow \infty$ such that, for $t_{j}=t\left(\gamma\left(\tau_{j}\right)\right)$, the spheres $S_{\gamma}\left(t_{j}\right) \subset A_{\gamma}\left(\tau_{j}\right)$ satisfy $S_{\gamma}\left(t_{j}\right)=S_{E}\left(t_{j}\right)$, i.e. the spheres $S_{E}\left(t_{j}\right)$ are connected, and hence $A_{\gamma}\left(\tau_{j}\right)=A_{E}\left(\tau_{j}\right)$. Thus, $u$ becomes uniformly unbounded on $A_{E}\left(\tau_{j}\right)$, as $j \rightarrow \infty$. However, as $\tau \rightarrow-\infty$, the curve $\gamma(\tau)$ also diverges to infinity in $E$ and $u(\gamma(\tau))$ is decreasing, (and so in particular bounded), as $\tau \rightarrow-\infty$. This contradiction implies that $u(\gamma(\tau)) \rightarrow u^{+}<+\infty$, as $\tau \rightarrow+\infty$. Of course $u^{+}>0$.

Suppose first, (for simplicity), that $\limsup _{E} u=u^{+}$, i.e. $\lim _{t \rightarrow \infty} m(t)=u^{+}$, where $m(t)=$ $\sup _{S_{E}(t)} u$. The maximum principle for the harmonic function $u$ implies that for $t$ sufficiently large, the function $m(t)$ is either monotone increasing or monotone decreasing in $t$ and hence approaches the value $u^{+}$as $t \rightarrow \infty$. Consider the annuli $A_{\gamma}(\tau)$ in the scale $g_{t}=t^{-2} \cdot g, t=t(\gamma(\tau))$, as in (3.5). Any sequence $\tau_{i} \rightarrow \infty$ has a subsequence such that the corresponding annuli $\left(A_{\gamma}\left(\tau_{i}\right), g_{t_{i}}\right)$ converge to a limiting domain $A_{\infty}\left(\frac{1}{2}, 2\right)$ in a tangent cone at infinity $\left(A_{\infty}, g_{\infty}\right)$, or $\left(\bar{A}_{\infty}, g_{\infty}\right)$, passing to covers as described above in the case of collapse. By construction, we then see that the potential function $u_{\infty}$ for this limit static vacuum solution achieves its maximal value $u^{+}>0$ at an interior point. Since $u_{\infty}$ is harmonic, the maximum priniple implies that $u_{\infty} \equiv u^{+}$, and hence by the vacuum equations $(0.1)$, the limit $\left(A_{\infty}, g_{\infty}\right)$ or $\left(\bar{A}_{\infty}, g_{\infty}\right)$ is flat. This argument holds for any subsequence, and since the convergence to the limit is smooth, we see that

$$
\left.t^{2} \cdot r\right|_{A_{\gamma}\left(\frac{1}{2} t, 2 t\right)} \rightarrow 0, \quad t=t(\gamma(\tau)), \text { as } \tau \rightarrow+\infty,
$$

by the scale-invariance of this expression.

It follows from Lemma 3.2 and Remark 3.3(iii) that the end $E$ is topologically standard, and the annuli $A_{E}\left(\frac{1}{2} t, 2 t\right), t=t(\gamma(\tau))$, are connected in $E$, for all $\tau$ sufficiently large. From the prior argument, this implies in particular that $u \rightarrow u^{+}$uniformly at infinity in $E$.

However, as before, as $\tau \rightarrow-\infty, u(\gamma(\tau))$ is monotone decreasing to a value $u_{-} \geq 0$. It follows that $u^{+}=u_{-}$. This is of course impossible, and shows that $\gamma(\tau)$ must have exited $E$ at some (negative) time.

Thus it remains to prove that $L \equiv \limsup _{E} u=u^{+}$. Since the annuli $A_{E}\left(\tau_{j}\right)$ above are connected, the Harnack inequality (3.4), together with the fact that $u^{+}<\infty$, implies that $L<\infty$. Now choose points $x_{j} \in S_{\gamma}\left(t_{j}\right)$ such that $u\left(x_{j}\right) \rightarrow L$. As above, the functions $\left.u\right|_{A_{E}\left(\tau_{j}\right)}$ have a subsequence converging to a limit harmonic function $u_{\infty}$ on a tangent cone at infinity based at $x_{\infty}=\lim x_{j}$. Then as before $u$ has an interior maximum at $x_{\infty}$ and hence $u_{\infty} \equiv L$; this gives $L=u^{+}$.

It follows from Lemma 3.4 and the discussion preceding it that any maximal flow line of $\nabla u$ intersects an apriori given large compact set $K \subset M$, except those exceptional flow lines which start or end at a critical point of $u$ far out in $M$. In particular, a set of full measure in any given level set $L$ of $u$ may be connected to points in $K$ by flow lines of $\nabla u$. In this sense, $u$ is 'almost proper', in that it behaves almost like a proper function in terms of the gradient flow.

Observe that this does not necessarily imply that the level sets of $u$ are compact, i.e. that $u$ is proper. For instance, the Weyl solution generated by the dipole potential $\nu=\nu_{+}+\nu_{-}$considered 
in $\S 2(\mathrm{IIC})$ satisfies (3.6), (it is even asymptotically flat), but the 0-level of $\nu$ is non-compact if $\nu_{+}$ and $\nu_{-}$are chosen so that the mass is 0 . In this example, the only divergent flow lines of $\nabla u$ are the two ends of the $z$-axis.

Next, as in $\S 2$, let

$$
\nu=\log u
$$

The following result is quite standard.

Lemma 3.5. On $\left(N, g_{N}\right)$ as in (0.2), with Riemannian metric, we have

$$
\Delta_{N}|\nabla \nu| \geq 0 \text {. }
$$

Proof: This standard estimate is a simple consequence of the Bochner-Lichnerowicz formula

$$
\frac{1}{2} \Delta|\nabla \nu|^{2}=\left|D^{2} \nu\right|^{2}+<\nabla \Delta \nu, \nabla \nu>+r(\nabla \nu, \nabla \nu),
$$

on $\left(N, g_{N}\right)$, where we have dropped the subscript $N$ from the notation. Since $\Delta \nu=0$, by (1.6), and since $\left(N, g_{N}\right)$ is Ricci-flat, this gives

$$
\frac{1}{2} \Delta|\nabla \nu|^{2} \geq\left|D^{2} \nu\right|^{2}
$$

One computes

$$
\Delta|\nabla \nu|=\frac{1}{2}|\nabla \nu|^{-1} \Delta|\nabla \nu|^{2}-\left.\left.\frac{1}{4}|\nabla \nu|^{-3}|\nabla| \nabla \nu\right|^{2}\right|^{2},
$$

and, by the Cauchy-Schwarz inequality $\left.\left.|\nabla| \nabla \nu\right|^{2}\right|^{2} \leq 4\left|D^{2} \nu\right|^{2}|\nabla \nu|^{2}$, so the result follows.

Lemmas 3.4 and 3.5 lead to the following key result relating the behavior of $|\nabla u|$ to the area growth of geodesic spheres. This result is a straightforward consequence of the divergence theorem for proper harmonic functions $u$ on manifolds of non-negative Ricci curvature. Lemma 3.4 allows one to remove the assumption that $u$ is proper.

Lemma 3.6. There is a constant $C<\infty$ such that for any component $S_{c}(s)$ of $S(s) \subset M, s \geq 1$,

$$
\sup _{S_{c}(s)}|\nabla u| \leq C \cdot \operatorname{area} S_{c}(s)^{-1} .
$$

Proof. We work on the Riemannian 4-manifold $\left(N, g_{N}\right)$ until the end of the proof. Let $\hat{A}_{c}=$ $\hat{A}_{c}(s)=\pi^{-1}\left(A_{c}\left(\frac{1}{2} s, 2 s\right)\right)$ and $\hat{S}_{c}=\hat{S}_{c}(s)=\pi^{-1}\left(S_{c}(s)\right)$, where $\pi: N \rightarrow M$ is projection on the first factor, with $S^{1}$ fibers. From the coarea formula, we have

$$
\int_{\hat{A}_{c}}|\nabla \nu|^{2}=\int_{v} \int_{L_{v} \cap \hat{A}_{c}}|\nabla \nu| d \sigma_{v} d v
$$

where $L_{v}$ is the $v$-level set of $\nu$ in $N$ and the outer integral in (3.9) is over the range of values in $\mathbb{R}$ of $\nu$ in $\hat{A}_{c}$. Now as remarked following Lemma 3.4, up to a set $\hat{Z}_{v}$ of measure 0 in $L_{v} \cap \hat{A}_{c}$, all points in the set $\left(L_{v} \cap \hat{A}_{c}\right) \backslash \hat{Z}_{v}$ may be joined by flow lines of $\nabla \nu$ to points in a fixed bounded hypersurface $\hat{T}$ in $\hat{K}$, independent of $v, s$; here $K$ is the compact set from Lemma 3.4 , and $\hat{T}=\partial \hat{K}$ for instance. Hence, by the divergence theorem applied to the harmonic function $\nu$ on $N$,

$$
\int_{L_{v} \cap \hat{A}_{c}}|\nabla \nu| \leq \int_{\hat{T}}|\nabla \nu| \leq c_{1},
$$

for some $c_{1}<\infty$, independent of $s$ and $\hat{A}_{c}$.

Now $|\nabla \nu|$ is subharmonic on $\left(N, g_{N}\right)$ by Lemma 3.5 , and $\operatorname{diam}_{N}^{i} \hat{A}_{c} \leq c \cdot s$, by (the proof of) Lemma 3.1. A standard sub-mean value inequality for manifolds of non-negative Ricci curvature, c.f. [SY,Thm.II.6.2], then gives

$$
\sup _{\hat{S}_{c}(s)}|\nabla \nu|^{2} \leq \frac{c_{2}}{\operatorname{vol} \hat{A}_{c}} \int_{\hat{A}_{c}}|\nabla \nu|^{2}
$$


Hence the estimates (3.9)-(3.11) imply

$$
\sup _{\hat{S}_{c}(s)}|\nabla \nu|^{2} \leq c_{3} \cdot \operatorname{osc}_{\hat{A}_{c}} \nu \cdot\left(\operatorname{vol} \hat{A}_{c}\right)^{-1} .
$$

To estimate the right hand side of (3.12), again by (the proof of) Lemma 3.1, osc $\nu \leq c_{4} \cdot \sup |\nabla \nu| s$ on $\hat{A}_{c}$. Further, we claim that

$$
\sup _{\hat{A}_{c}(s)}|\nabla \nu| \leq \operatorname{csup}_{\hat{S}_{c}(s)}|\nabla \nu|,
$$

for some constant $c$ independent of $s$ and $\hat{A}_{c}$. To see this, by scale-invariance, it suffices to prove that $\sup _{\hat{A}_{c}(1)}|\nabla \nu| \leq \operatorname{csup}_{\hat{S}_{c}(1)}|\nabla \nu|$ w.r.t. the rescaled metrics $g_{s}=s^{-2} g$. By the curvature and diameter bounds (3.3) and (3.4) and Lemmas 1.3 and 1.4, the metrics $\left(\hat{A}_{c}(1), g_{s}\right)$ form a compact family of metrics in the $C^{\infty}$ topology, unwrapping in the case of collapse. Thus, one has uniform control on the metrics $g_{s}$ on $\hat{A}_{c}(1)$. Similarly, when normalized if necessary by additive and multiplicative constants so that $\sup _{\hat{S}_{c}(1)} \nu=\sup _{\hat{S}_{c}(1)}|\nabla \nu|=1$, the positive harmonic functions $\nu$ on $\left(\hat{A}_{c}(1), g_{s}\right)$ also form a compact family of functions in the $C^{\infty}$ topology, i.e. a normal family. This follows by the Harnack estimate (3.4) and the Harnack principle (elliptic regularity) for harmonic functions, c.f. [GT, Thm 2.11, Ch. 8]. This compactness of the metrics and functions from elliptic theory proves the claim above.

Similarly, the metric compactness above also implies there is a constant $c<\infty$ such that

$$
c^{-1} \cdot \operatorname{area} S_{c}(1) \leq \operatorname{vol} A_{c}(1) \leq c \cdot \operatorname{area}_{c}(1),
$$

w.r.t. the metrics $g_{s}$. (This estimate can also be derived directly from the Bishop-Gromov volume comparison theorem). Rescaling back to the metric $g$ then gives

$$
c_{5}^{-1} \cdot s \cdot \operatorname{area} S_{c}(s) \leq \operatorname{vol} A_{c} \leq c_{5} \cdot s \cdot \operatorname{area} S_{c}(s),
$$

for some constant $c_{5}<\infty$. Note that by definition,

$$
\operatorname{area} \hat{S}_{c}(s)=\int_{S_{c}(s)} u d A
$$

and the same for $\operatorname{vol} \hat{A}_{c}$. Hence by (3.4), the estimate (3.13) holds also for $\hat{S}_{c}(s)$ and $\hat{A}_{c}$ in place of $S_{c}(s)$ and $A_{c}$.

Thus, by combining these estimates above, (3.12) gives

$$
\sup _{\hat{S}_{c}(s)}|\nabla \nu| \leq c_{6} \cdot \operatorname{area} \hat{S}_{c}(s)^{-1} .
$$

Using (3.14) and (3.4) again, this estimate implies (3.8).

We are now in a position to begin the proof of Theorem 0.3 itself. Observe that the previous results in $\S 3$ have not used the assumption (3.2), nor the assumption (ii) in Theorem 0.3 that $u$ does not approach 0 everywhere at infinity in $E$. Only the assumption that $\partial M$ is pseudo-compact has been used. Hence, at this stage, we do not even know that $E$ has finite topological type. The main point initially is to prove that the estimates (3.6)-(3.7) above do hold on $E$ under these assumptions.

Recall that we have $S_{E}(s)=\cup S_{c}(s)$, for $S_{c}(s) \subset E$. Each geodesic ray $\sigma(s)$ in $E$, i.e. an integral curve of $\nabla t$, with $\sigma(s) \in S_{E}(s)$, determines a component $S_{\sigma}(s)=S_{c}(s)$ s.t. $\sigma(s) \in S_{c}(s)$; the union of such components sweep out a part $E_{\sigma}$ of the end $E$. Of course $E$ is the union of $E_{\sigma}$ among all (non-homotopic) rays $\sigma$.

From Lemma 3.1 and from the obvious area $S_{E}(s)=\sum \operatorname{area} S_{c}(s)$, we have

$$
\int^{\infty} \operatorname{areaS}_{E}(s)^{-1} d s<\infty \Leftrightarrow \int^{\infty} \operatorname{area} S_{\sigma}(s)^{-1} d s<\infty
$$


for some geodesic ray $\sigma \subset E$. (Here the integrals start at some fixed value $s \geq s_{o}>0$ ).

Hence, under the assumption (3.2), we have

$$
\int^{\infty} \operatorname{area} S_{\sigma}(s)^{-1} d s \leq K<\infty
$$

for some ray $\sigma \subset E$ and constant $K$.

By integrating along the curve $\sigma,(3.15)$, Lemma 3.6 and the Harnack estimate (3.4) imply that $u$ is uniformly bounded in $E_{\sigma}$. In fact, we claim that

$$
u_{\infty}=\lim _{t(x) \rightarrow \infty} u(x)<\infty
$$

exists, where the limit is taken in $E_{\sigma}$. To see this, let $\gamma(s)$ be any "quasi-geodesic" in $E_{\sigma}$, i.e. $\gamma$ is a smooth curve with $\gamma(s) \in S_{\sigma}(s)$ and $|d \gamma / d s| \leq C_{1}$, for some $C_{1}<\infty$. By (3.8) and (3.15), we then have $\int^{\infty}|d u(\gamma(s))| d s \leq C \cdot C_{1} \cdot K<\infty$, and so $u\left(\gamma\left(s_{1}\right)\right)-u\left(\gamma\left(s_{2}\right)\right) \rightarrow 0$ whenever $s_{1}, s_{2} \rightarrow \infty$. Hence the limit $u_{\infty}(\gamma)$ is well-defined. The diameter estimate (3.1) implies that all points in $E_{\sigma}$ lie on quasi-geodesics, (with a fixed $C_{1}$ ), in $E_{\sigma}$, starting on $S_{\sigma}\left(s_{o}\right)$. Further, the limit $u_{\infty}(\gamma)$ is clearly independent of $\gamma$, since for instance (3.8) and (3.15) imply that

$$
\operatorname{osc}_{A_{\sigma}\left(\frac{1}{2} s_{j}, 2 s_{j}\right)} u \rightarrow 0
$$

on some sequence $s_{j} \rightarrow \infty$. Hence (3.16) follows.

Next, since $E$ is an end, there exists some sequence $t_{j} \rightarrow \infty$ such that the geodesic spheres $S_{E}\left(t_{j}\right)$ are connected, and hence $S_{\sigma}\left(t_{j}\right)=S_{E}\left(t_{j}\right)$. By $(3.16),\left.u\right|_{S_{E}\left(t_{j}\right)} \rightarrow u_{\infty}$ as $t_{j} \rightarrow \infty$. The maximum principle applied to the harmonic function $u$ thus implies that $\left.u\right|_{A_{E}\left(t_{j}, t_{k}\right)} \rightarrow u_{\infty}$, whenever $t_{j}, t_{k} \rightarrow \infty$. Thus, we see that (3.16) holds where the limit is taken in the full end $E$, and not just in $E_{\sigma}$.

Now we use the assumption (ii) of Theorem 0.3 , which says that $u\left(x_{j}\right) \geq u_{o}>0$, for some constant $u_{o}>0$ and some divergent sequence $x_{j} \in E$. It follows from this and the existence of the limit (3.16) in $E$ that

$$
u_{\infty}>0 .
$$

Hence, we may, and will, renormalize the potential function $u$ of the static vacuum solution $(M, g, u)$ so that, on $E$,

$$
\lim _{t(x) \rightarrow \infty} u(x)=1 .
$$

The estimate (3.17) essentially immediately implies the scale-invariant estimates

$$
\sup _{S_{E}(s)}|r|<<s^{-2}, \sup _{S_{E}(s)}|\nabla u|<<s^{-1} \text {, as } s \rightarrow \infty,
$$

strenthening the bounds (3.3). For as discussed following (3.3), (3.18) is equivalent to the statement that all tangent cones at infinity of $E$ are flat, (c.f. also the proof of Lemma 3.2). But, as noted above, all tangent cones at infinity are static vacuum solutions, and (3.17) implies that the limit potential $u_{\infty}$ satisfies $u_{\infty} \equiv 1$. Hence, the static vacuum equations (0.1) of course imply the limit metrics are flat.

Lemma 3.2 now determines the topology of the end $E$, as one of two (standard) alternatives, according to non-collapse or collapse behavior at infinity.

Before proceeding to the analysis of these cases, note that (3.18) implies that the metrics $g$ and $\tilde{g}=u^{2} \cdot g$ from (1.4) are quasi-isometric on $E$, and almost isometric near infinity. Since $\tilde{g}$ has non-negative Ricci curvature on $E$, standard volume comparison theory implies that the area and volume ratios

$$
\frac{\operatorname{area}_{\tilde{g}}(\tilde{S}(s))}{s^{2}}, \frac{\operatorname{vol}_{\tilde{g}}(\tilde{B}(s))}{s^{3}}
$$


are monotone non-increasing in $s$. Hence, their limits at $s=\infty$ exist, and by the equality of $g$ and $\tilde{g}$ at infinity, the limits at $s=\infty$ of the ratios in (3.19) w.r.t. the $g$ metric and $g$-geodesic spheres and balls also exist, and equal the $\tilde{g}$ limits.

To proceed further, we now separate the discussion into non-collapse and collapse cases.

\section{Case A. (Non-Collapse).}

Suppose that $E$ is non-collapsing at infinity, i.e. by the remarks above,

$$
\limsup _{s \rightarrow \infty} \frac{v(s)}{s^{3}}=\lim _{s \rightarrow \infty} \frac{v(s)}{s^{3}}>0,
$$

where $v(s)$ denotes $\operatorname{vol}_{g}\left(B_{E}(s)\right)$. This implies, via (3.13), that areaS(s) ${ }^{-1} \leq c \cdot s^{-2}$, and hence, by Lemma 3.6,

$$
\sup _{S(s)}|\nabla u| \leq c s^{-2} .
$$

The volume condition (3.20) implies that all tangent cones at infinity $A_{\infty}$ of $(E, g)$ exist, without passing to covering spaces, and, as discussed above, are flat solutions of the static vacuum equations with potential $u_{\infty} \equiv 1$. As discussed in the proof of Lemma 3.2, the tangent cone at infinity is unique, (up to isometry), and given by $\mathbb{R}^{3} \backslash\{0\}$, and further $E \backslash K$ is diffeomorphic to $\mathbb{R}^{3} \backslash B$, for some compact set $K \subset E$. The blow-down metrics $g_{s}=s^{-2} \cdot g$ on all annuli $A_{E}\left(\frac{1}{2} s, 2 s\right)$ converge smoothly to the flat metric on $A(1,2) \subset \mathbb{R}^{3}$, uniformly as $s \rightarrow \infty$, and hence there are local (harmonic) coordinates on $A_{E}\left(\frac{1}{2} s, 2 s\right)$ in which $g$ has the expansion $g_{i j}=\delta_{i j}+\gamma_{i j}$, where $\left|\gamma_{i j}(x)\right| \rightarrow$ 0 uniformly as $s \rightarrow \infty$. Again as discussed in the proof of Lemma 3.2, these local coordinates, e.g. on $\left\{A_{E}\left(2^{i-1}, 2^{i+1}\right)\right\}, i>0$, may be assembled into a global chart, mapping $E \backslash K$ onto $\mathbb{R}^{3} \backslash B$, c.f. $[\mathrm{AC}]$ for further details if desired. With respect to such a chart, the metric $g_{i j}$ has the form

$$
g_{i j}=\delta_{i j}+\gamma_{i j}
$$

on all of $E \backslash K$, with $\left|\gamma_{i j}\right| \rightarrow 0$ uniformly at infinity in $E$. In other words, $g$ is $C^{o}$ asymptotic to the flat metric at infinity.

To prove that the metric on $E$ is (strongly) asymptotically flat, as defined preceding Theorem 0.1 , consider again the metric $\tilde{g}=u^{2} \cdot g$. From (1.4) and (3.21), the curvature of $\tilde{g}$ decays as

$$
|\tilde{r}| \leq C t^{-4}
$$

as $t \rightarrow \infty$. (Note also that $t$ and $\tilde{t}$ are approximately equal for $t$ large).

It follows that the expansion (3.22) may be improved, for $\tilde{g}$, to

$$
\tilde{g}_{i j}=\delta_{i j}+O\left(t^{-2}\right),
$$

in a suitable (harmonic) coordinate chart. We refer to $[\mathrm{BKN}]$ or $[\mathrm{BM}]$ for instance for further details here. Briefly, elliptic regularity theory applied to the equations (1.4)-(1.5), together with the curvature decay above, implies that the $2^{\text {nd }}$ derivatives of the metric $\tilde{g}$ in the coordinate chart decay as $t^{-4}$, so that the metric $\tilde{g}$ decays to the flat metric at a rate of $t^{-2}$. Hence

$$
g_{i j}=u^{-2} \tilde{g}_{i j}=(1+2 v) \delta_{i j}+O\left(t^{-2}\right),
$$

where $v=1-u$. Here we are using that fact that since $|\nabla u|=O\left(t^{-2}\right), u$ has an expansion of the form $u=1+O\left(t^{-1}\right)$. Further, since $\log u$ is harmonic w.r.t. $\tilde{g}$, the decay (3.21) and (3.23) implies that $\Delta_{f} \log u=O\left(t^{-4}\right)$ for $t$ large, where $\Delta_{f}$ is the flat Laplacian on $\mathbb{R}^{3}$. This means that $u$ has an expansion $u=1-\frac{m}{t}+O\left(t^{-2}\right)$, where $m$ is the mass of $E$ defined in (0.7).

In particular, these estimates show that the end $E$ is asymptotically flat in the sense preceding Theorem 0.1.

Note that, to first order in $t^{-1}$, the function $v=1-u$ corresponds to the Green's function in $\mathbb{R}^{3}$, i.e. the fundamental solution of the Laplacian, weighted by the mass $m$. It is of course possible to have $m=0$, as for instance for the dipole-type Weyl solutions in $\S 2$ (IIC), or also $m<0$. Further, 
since $u$ has been normalized so that $u \rightarrow 1$ at infinity in $E$, the expression (0.7) for the mass is equivalent to the usual definition

$$
m_{E}=\frac{1}{4 \pi} \int_{S_{E}(s)}<\nabla u, \nabla t>d A
$$

where $s$ is sufficiently large so that $S_{E}(s) \cap \partial E=\emptyset$. This is because the expression (3.25) is independent of $s$, since $u$ is harmonic, and the fact that it is asymptotic to the expression (0.7) as $s \rightarrow \infty$.

This completes the analysis of Case A.

Case B. (Collapse).

Under the standing assumption (3.15), suppose that the end $E$ is collapsing at infinity, i.e.

$$
\limsup _{s \rightarrow \infty} \frac{v(s)}{s^{3}}=\lim _{s \rightarrow \infty} \frac{v(s)}{s^{3}}=0 .
$$

We will prove that this situation is impossible. The results preceding Case A remain valid, so that (3.17)-(3.18) hold, all tangent cones at infinity $\bar{A}_{\infty}$ of $E$ are flat products of the form $V \times S^{1}$ or $\mathbb{R}^{+} \times S^{1} \times S^{1}$, where $V$ is a flat 2-dimensional cone. Further $E \backslash K$ is diffeomorphic to $\left(\mathbb{R}^{2} \backslash B\right) \times S^{1}$, for some compact set $K \subset E$.

By (3.15) and Lemma 3.6, we have

$$
\int^{\infty} \sup _{S(s)}|\nabla u|(s) d s \leq K_{1}<\infty .
$$

The main point is now to show that $(E, g)$ itself, (and not just its tangent cones), is asymptotic to a flat quotient of $\mathbb{R}^{3}$, and hence has at most quadratic volume growth of geodesic balls or linear area growth of geodesic spheres. This is done in the following result, which is a strengthening of Lemma 3.2.

Lemma 3.7. Under the assumptions (3.26) and (3.27) above, there is a compact set $K \subset E$ such that $(E \backslash K, g)$ is quasi-isometric to a flat product $\left(\mathbb{R}^{2} \backslash B\right) \times S^{1}$ or $\mathbb{R}^{+} \times S^{1} \times S^{1}$.

Proof: As in Case A, it is useful to work with the metric $\tilde{g}=u^{2} g$; again, this makes no significant difference, since (3.17) holds. All the metric quantities below are thus in the $\tilde{g}$ metric. For notational simplicity however, we drop the tilde from the notation.

Let $t_{\infty}(x)=\lim _{s \rightarrow \infty}\left(\operatorname{dist}\left(x, S_{E}(s)\right)-s\right)$. As in the construction of Busemann functions, the limit here exists, c.f. [Wu] for a discussion of such functions. By construction, $t_{\infty}$ is a Lipschitz distance function, i.e. $t_{\infty}$ realizes everywhere the distance between its level sets. Observe that on $\mathbb{R}^{3}, t_{\infty}$ is just the distance function to $\{0\} \in \mathbb{R}^{3}$, on $V \times S^{1}, t_{\infty}$ is the distance function to $\{0\} \in V$ pulled back to $V \times S^{1}$, for any cone $V$ with vertex $\{0\}$, while on $\mathbb{R}^{+} \times S^{1} \times S^{1}, t_{\infty}$ is the distance function on $\mathbb{R}^{+}$pulled back to the total space.

By renormalization, (as with the potential $u$ ), $t_{\infty}$ induces a distance function $\bar{t}_{\infty}$ on each tangent cone $\bar{A}_{\infty}$ by defining $\bar{t}_{\infty}(x)=\lim \left(t_{\infty}(x) / t_{\infty}\left(x_{i}\right)\right)$, where $t\left(x_{i}\right) \rightarrow \infty$ and $x_{i}$ are the base points converging to the base point $x_{\infty} \in \bar{A}_{\infty}$. Thus $\bar{t}_{\infty}$ is the function above on $V \times S^{1}$ or $\mathbb{R}^{+} \times S^{1} \times S^{1}$.

The map $t_{\infty}:(E, \tilde{g}) \rightarrow \mathbb{R}^{+}$is distance non-increasing, and preserves distance along the integral curves of $t_{\infty}$; thus where smooth, it is a Riemannian submersion. We will show that $t_{\infty}$ gives rise to a Lipschitz quasi-isometry by examining the asympotics of the second fundamental form of its level sets.

Thus, let $\sigma(s)$ be any geodesic ray in $E$ which is an integral curve of $\nabla t_{\infty}$, and let $B=B_{\sigma}(s)$ denote the second fundamental form of the level surface $t_{\infty}^{-1}(s)$ at $\sigma(s)$. The form $B$ is well-defined and smooth along any such ray $\sigma$. Recall that $B$ satisfies the Riccati equation

$$
B^{\prime}+B^{2}+R_{T}=0
$$


where $T$ is the unit tangent vector along $\sigma$. Consider the behavior of $s \cdot B(s)$ as $s \rightarrow \infty$. This quantity is scale-invariant, and thus converges smoothly, (in subsequences), to the limiting expression $\bar{s}$. $B_{\infty}(\bar{s})$ on any tangent cone at infinity. Since the parameters $s$ and $\left.t_{\infty}\right|_{\sigma}$ are the same up to additive constants, $\bar{s}=\bar{t}_{\infty}$. Similarly, by the definition of $\bar{t}_{\infty}, B_{\infty}$ is the second fundamental form of the levels $\bar{t}_{\infty}$. Hence, either

(i): $\bar{s} \cdot B_{\infty}(\bar{s})=(d \theta /|d \theta|)^{2}$, when the tangent cone is of the form $V \times S^{1}$, and $\theta$ is the angle variable about $\{0\} \in V$, or

(ii): $\bar{s} \cdot B_{\infty}(\bar{s})=0$, when the tangent cone is of the form $\mathbb{R}^{+} \times S^{1} \times S^{1}$.

Thus $\bar{s} \cdot B_{\infty}(\bar{s})$ is either of rank 1 , with eigenvalue 1 , or identically 0 . Note that the expression in case (i) is independent of the cone $V$, i.e. the cone angle at $\{0\}$.

As noted preceding Lemma 3.2 and in Remark 3.3(ii), the geometry of $\bar{A}\left(\frac{1}{2} s, 2 s\right)$ smoothly approximates that of a limit tangent cone $\bar{A}_{\infty}$, for $s$ large, and varies continuously in $s$. Since the two alternatives (i) and (ii) above for the structure of $B_{\infty}$ are rigid, it follows that all tangent cones $\bar{A}_{\infty}$ are of the same type, i.e. they are all of the form $V \times S^{1}$, or all of the form $\mathbb{R}^{+} \times S^{1} \times S^{1}$.

The main task now is to show that the deviation of $s \cdot B(s)$ from its limit $\bar{s} \cdot B_{\infty}(\bar{s})$ has bounded integral. To do this, we use the Riccati equation, and estimate the decay of the curvature term $R_{T}$, using basically standard methods in comparison geometry, c.f. [P, Ch.6.2] for instance.

Thus, from (1.4), the sectional curvature $K$ of $(M, \tilde{g})$ satisfies

$$
K_{X Z}=|\nabla \nu|^{2} \geq 0, \quad K_{X Y}=-|\nabla \nu|^{2} \leq 0
$$

where $Z=\nabla u /|\nabla u|, X, Y$ are vectors orthogonal to $Z$, and $\nu=\log u$. Hence $\left|R_{T}\right| \leq|\nabla \nu|^{2}$. Substituting this in (3.28) gives

$$
\left|B^{\prime}+B^{2}\right| \leq|\nabla \nu|^{2}
$$

Let $\lambda$ be any eigenvalue of $B$, with unit eigenvector $e$; (note that $B$ is symmetric). Observe then that $s \cdot \lambda(s)$ converges either to 1 or to 0 , as $s \rightarrow \infty$. The estimate (3.29) when applied to $(e, e)$ gives

$$
\pm\left(s \lambda^{\prime}+s \lambda^{2}\right) \leq s|\nabla \nu|^{2}
$$

Integrate this by parts along any finite interval $I$ to obtain

$$
|s \lambda|_{\partial I}+\left.\int_{I}\left(s \lambda^{2}-\lambda\right) d s\left|\leq \int_{I} s\right| \nabla \nu\right|^{2} d s
$$

If $s \lambda \rightarrow 0$ as $s \rightarrow \infty$, choose $I$ to be any interval on which $s \lambda=0$ at $\partial I$ and $s \lambda \neq 0$ on $I$, so that $s \lambda$ has a definite sign on $I$. If there are no such boundary points, choose $I$ to be an infinite half-line. Similarly, if $s \lambda \rightarrow 1$, choose $I$ to be intervals such that $s \lambda=1$ at $\partial I$ with $s \lambda-1 \neq 0$ on $I$. Then summing up the estimate above over all such intervals gives

$$
\int_{\sigma}\left|s \lambda^{2}-\lambda\right| d s \leq \int_{\sigma} s|\nabla \nu|^{2} d s+C_{o},
$$

for some constant $C_{o}<\infty$. Now the estimate (3.18), together with (3.27) gives

$$
\int s|\nabla \nu|^{2} d s \leq C_{1}
$$

for some constant $C_{1}<\infty$.

Suppose first $s \cdot \lambda(s) \rightarrow 1$ as $s \rightarrow \infty$. We then obtain

$$
\int\left|s \lambda\left(\lambda-\frac{1}{s}\right)\right| d s \leq C_{2}<\infty
$$

and hence

$$
\int\left|\lambda-\frac{1}{s}\right| d s \leq C_{3}<\infty
$$


Similarly, if $s \cdot \lambda(s) \rightarrow 0$ as $s \rightarrow \infty$, one obtains

$$
\int|\lambda| d s \leq C_{3}<\infty
$$

Now the second fundamental form $B$ gives the logarithmic derivative of the norm of Jacobi fields formed by the family of $t_{\infty}$-rays in $E$ starting at some level $t_{\infty}^{-1}\left(s_{o}\right)$. Thus, if $J$ is any Jacobi field formed from the $t_{\infty}$-conguence, and $v=J /|J|$, we have along any $t_{\infty}$-ray,

$$
B(v, v)=\frac{d}{d s}(\log |J|(s)) .
$$

Suppose first that the end $E$ has tangent cones at infinity of the form $\mathbb{R}^{+} \times S^{1} \times S^{1}$. Then (3.31) implies the uniform bound

$$
C_{4}^{-1} \leq|J|(s) \leq C_{4},
$$

with $C_{4}=e^{C_{3}}$. This means that the geometry of the level surfaces of $t_{\infty}$ is uniformly bounded as $s \rightarrow \infty$, i.e. the diameter and area of the level surfaces is uniformly bounded away from 0 and $\infty$. It is then clear that there is a Lipschitz quasi-isometry of $(E \backslash K, \tilde{g})$ to $\mathbb{R}^{+} \times S^{1} \times S^{1}$ induced by $t_{\infty}$. Since $\tilde{g}$ and $g$ are also quasi-isometric, by (3.17), the lemma follows in this case.

If $E$ has tangent cones at infinity of the form $V \times S^{1}$, then there is a basis of Jacobi fields whose elements satisfy either (3.32), or, from (3.30),

$$
C_{4}^{-1} s \leq|J|(s) \leq C_{4} s .
$$

As before, this implies that $t_{\infty}$ gives rise to a quasi-isometry of $(E \backslash K, g)$ to $\left(\mathbb{R}^{2} \backslash B\right) \times S^{1}$.

Of course Lemma 3.7, in both cases, immediately implies that

$$
\operatorname{area}_{E}(s) \leq c \cdot s,
$$

for some constant $c<\infty$. However, (3.34) violates the standing assumption (3.15), (or (3.2)). It follows that no end $(E, g)$ can satisfy the assumptions of Case B.

Together with Case A, this completes the proof of the second statement in Theorem 0.3.

We now turn to the proof of the last statement in Theorem 0.3. We will assume that the end $E$ is small, i.e.

$$
\int_{E} \frac{1}{\operatorname{areaS} S_{E}(s)} d s=\infty
$$

and derive a contradiction from the assumptions supu $<\infty$ and $m_{E} \neq 0$.

The proof is based on a result of Varopoulos [V] which states that ends of Riemannian manifolds satisfying (3.35) are parabolic, i.e. admit no non-constant positive superharmonic functions $v$ which tend uniformly toward their infimum at infinity. (Actually, the result in $[\mathrm{V}]$ is a condition on the volume growth of geodesic balls, but this is equivalent to the bound (3.35) under the estimate (3.13)). We will prove that the potential $u$ is such a non-constant function, giving the required contradiction.

Thus, suppose first that

$$
\sup _{E} u<\infty
$$

Arguing as in (3.9), but now on $E \subset M$ in place of $N$, we have

$$
\int_{E}|\nabla u|^{2}=\int_{v} \int_{L_{v} \cap E}|\nabla u| d A_{v} d v
$$


and as in (3.10),

$$
\int_{L_{v} \cap E}|\nabla u| \leq \int_{T}|\nabla u| \leq C .
$$

Thus, these estimates imply that

$$
\int_{E}|\nabla u|^{2}<\infty
$$

so that

$$
\int_{A_{E}(s, \infty)}|\nabla u|^{2} \rightarrow 0 \text { as } s \rightarrow \infty .
$$

On the other hand, again referring to the proof of Lemma 3.6, since almost all (in terms of measure) points in $L_{v} \cap E$ may be joined by flow lines of $\nabla u$ to a fixed bounded surface $T$ in $K$, by the divergence theorem there is a subsurface $T^{\prime} \subset T$ such that

$$
\int_{L_{v} \cap E}|\nabla u| \geq \int_{T^{\prime}}|\nabla u| \geq c,
$$

for some constant $c>0$. It follows from (3.37)-(3.40) that

$$
\text { osc }_{A_{E}(s, \infty)} u \rightarrow 0 \text { as } s \rightarrow \infty .
$$

By assumption (ii) in Theorem 0.3, we may thus assume w.l.o.g. that

$$
\lim _{t \rightarrow \infty} u=1,
$$

in $E$. Thus, as noted in (3.18), $|r| \leq \varepsilon(t) / t^{2}$, where $\varepsilon(t) \rightarrow 0$ as $t \rightarrow \infty$, and so Lemma 3.2 holds on $E$.

Now choose a smooth approximation $S$ to a large geodesic sphere $S_{E}(s)$ with $S_{E}(s) \cap \partial E=\emptyset$, so that $S$ separates $E$ into two components, one being the outside containing the end $E$.

We claim that if, in addition to (3.36),

$$
m_{E} \neq 0,
$$

then there is a set of flow lines $\gamma$ of $\nabla u$ or $-\nabla u$, starting on $S$, of positive measure on $S$, and pointing out of $S$, which never intersects $S$ again at later times. Hence such $\gamma$ diverge to infinity in $E$, since up to a set of measure $0, \gamma$ does not terminate in a critical point of $u$. (Compare with the earlier discussion regarding divergent flow lines and Weyl dipole solutions concerning Lemma 3.4).

To see this, suppose instead that all flow lines say of $\nabla u$ which initially point out of $S$ eventually intersect $S$ again, with the exception of those terminating in critical points. Consider the measure

$$
d \mu=<\nabla u, \nu>d A,
$$

on $S$, where $d A$ is the Lebesgue measure and $\nu$ is the unit outward normal on $S$; $d \mu$ is absolutely continuous w.r.t. $d A$. Since $u$ is harmonic, the divergence theorem implies that the gradient flow of $u$ preserves the measure $d \mu$ in the following sense. Let $D$ be a domain in $S$ and let $\Omega$ be the domain in $E$ formed by a collection of flow lines outside $S$, whose endpoints form a smooth surface $D^{\prime}$. If $\nu^{\prime}$ denotes the unit outward normal to $\Omega$ at $D^{\prime}$, then the flow from $D$ to $D^{\prime}$ carries the measure $d \mu$ to the measure $d \mu^{\prime}=<\nabla u, \nu^{\prime}>d A^{\prime}$, where $d A^{\prime}$ is Lebesgue measure on $D^{\prime}$. In particular, the flow preserves the masses of the measures.

Thus, under the assumptions above, the gradient flow (with varying flow-times), induces a homeomorphism of $S \backslash Z$ into itself, where $Z$ is a set of Lebesgue measure 0, corresponding to flow lines terminating in critical points. However, this homeomorphism inverts the direction of $\nabla u$ w.r.t. the fixed normal $\nu$, and hence maps domains $D_{+}$on which the measure $d \mu$ is positive onto domains $D_{-}$on which $d \mu$ is negative, in such a way that that

$$
m_{\mu}\left(D_{+}\right)=\left|m_{\mu}\left(D_{-}\right)\right| \text {. }
$$


This of course implies that the total mass of $d \mu$ on $S$ is 0 . However using (3.44) and the remarks concerning (3.25), the mass $m_{E}$ of $E$ equals the total mass of $d \mu$. This contradiction proves the claim.

We may now complete the proof as follows. Assuming $E$ is an end satisfying (3.36) and (3.43), there exists an open set $\mathcal{O}$ of flow lines $\gamma=\gamma(\tau)$ of either $\nabla u$ or $-\nabla u$ which start on a set of positive measure on $S$ and diverge to infinity in $E$. Consider the former case, which corresponds to $m_{E}>0$. A generic flow line of $\nabla u$ in $E$ tends to the maximal value of $u$ in $E$ and hence a generic flow line in $\mathcal{O}$ also tends this maximal value. By (3.42), it then follows that $\sup _{E \backslash K} u=1$, for some compact set $K \subset E$. The function $v=-u$ is thus a bounded (non-constant) harmonic function on $E$, which tends uniformly to its infimum at infinity. Hence $E$ cannot be parabolic. This contradiction shows that (3.35) cannot hold for $E$, and thus, by the proofs in Cases A and B above, the end $E$ is asymptotically flat. The proof in case $m_{E}<0$ is the same.

This completes the proof of Theorem 0.3.

Remark 3.8.(i). There are (non-flat) static vacuum solutions with a small end, namely the Kasner metrics (2.33), with $a>0$. These solutions have $\operatorname{vol} B(s) \sim s^{2-\delta}, \operatorname{vol} S(s) \sim s^{1-\delta}$, where $\delta=\left(a+a^{-1}-1\right)^{-1} \in(0,1)$, and hence the end is small. Note that $u \sim s^{\delta}$ is unbounded. There are other Weyl solutions which are complete away from $\Sigma$ with $\partial M$ pseudo-compact and with one small end, (take for instance the potential given by the Green's function on $\mathbb{R}^{2} \times S^{1}$, see (ii) below), but all known examples with small ends are either asymptotic to the Kasner metric at infinity or have faster than quadratic curvature decay, i.e. satisfy (3.18).

It is an open problem to understand in more detail the structure of small ends of static vacuum solutions. It follows from the results above in $\S 3$ that all tangent cones at infinity of $E$ are collapsing, and hence they are all Weyl solutions. But the metric uniqueness of tangent cones at infinity is unknown, as is the question of whether small ends have finite topological type.

(ii). We construct an example which illustrates the sharpness of the last statement of Theorem 0.3. Let $G_{1}$ and $G_{2}$ be the Green's functions for the Laplacian on the flat product $\mathbb{R}^{2} \times S^{1}$, with poles at $\left(0, p_{i}\right)$, for $p_{1}, p_{2}$ distinct points in $S^{1}$. Here we consider $S^{1}$ as the $z$-axis in $\mathbb{R}^{3}$ quotiented out by an isometric $\mathbb{Z}$-action. As in $(2.34), G_{i}(x)=G\left(x, p_{i}\right)$, viewed as a function on the universal cover $\mathbb{R}^{3}$, may be written as

$$
G_{i}=\lim _{n \rightarrow \infty}\left[\sum_{j=-n}^{n} \frac{1}{r_{j}}-c_{n}\right],
$$

where $r_{j}$ is the Euclidean distance to the collection of lifts of $p_{i}$ in $\mathbb{R}^{3}$ and $c_{n}$ is a suitable normalizing constant with $c_{n} \rightarrow \infty$ as $n \rightarrow \infty$, chosen so that $G\left(x, p_{i}\right)$ is finite. Thus $G_{i}$ is an axisymmetric and $z$-periodic harmonic function on $\left(\mathbb{R}^{2}\right)^{+} \times S^{1}$, where the $S^{1}$ now means rotations about the $z$-axis in $\mathbb{R}^{3}$. Hence the potential

$$
\nu=G_{1}-G_{2}
$$

generates a Weyl solution as in (2.12), with $u=e^{\nu}$ and which has an isometric $\mathbb{Z}$-action along the $z$-axis. Let $M$ denote the $\mathbb{Z}$ quotient of this solution. Then the metric boundary of $M$ is pseudo-compact. Since $G_{i} \sim \log r$ as $r \rightarrow \infty$,

$$
u \rightarrow 1
$$

at infinity in $M$. The end $E=\mathbb{R}^{+} \times T^{2}$ is small and has mass 0 in the sense of (0.7). These solutions of course resemble the dipole-type solutions discussed in $\S 2$ (IIC), but with a collapsing end.

Remark 3.9.(i). Although we will not detail it here, an examination of the proof shows that Theorem 0.3 holds for non-vacuum static solutions of the Einstein field equations (1.2), provided suitable decay conditions are imposed on the stress-energy tensor $T$. 
This is the case for example, if $T$ is any tensor with compact support, or more generally if $T$ satisfies an estimate of the form $|T|(x) \leq c \cdot t^{-3}(x)$, for some $c>0$ and all $x$ with $t(x) \geq s_{o}$, together with $\frac{1}{u} \Delta u \in L^{1}(M \backslash U)$. This latter condition is needed to obtain the bound (3.10). By (1.2), note that $\frac{1}{u} \Delta u=\frac{1}{2} \operatorname{tr} T$. The starting estimate (3.3) may be obtained by applying (a suitable version of) [An1,Thm.3.3].

(ii). Also, Theorem 0.3 can be given a finite or quantitative formulation, i.e. one can relax the assumption of completeness, in basically the same way as the local estimates (3.3) follow from the non-existence of global static vacuum solutions with $\partial M=\emptyset$, c.f. [An1,App.].

Thus, if (3.15) holds and $(M, g)$ is 'sufficiently large', depending on $K$, then sufficiently far out in $(M, g)$, the metric is close to a flat metric. We leave a precise formulation and proof, (based on Theorem 0.3), to the reader.

Remark 3.10. As noted in $\S 0$, it would be of interest to prove that $(M, g, u)$ has a unique end. Under the hypotheses of Theorem 0.3 , we conjecture this is the case at least when $M$ is complete away from $\Sigma$. If $M$ is in addition smooth up to $\Sigma$, this has been proved by Galloway [G]. Following essentially the same arguments as in [G], it is not difficult to show that if $M$ is complete away from $\Sigma$ and if the Riemannian 4-manifold $N$ admits a compact smoothing of a neighborhood of $\Sigma$ having non-negative Ricci curvature, then $M$ has a unique end.

Remark 3.11. We point out that Theorem 0.3 and Theorem 0.1 are false in higher dimensions, due to the existence of Einstein metrics on compact manifolds, which are not of constant curvature, in dimensions $\geq 3$. (The equations $(0.1)$ on any $n$-dimensional manifold $M^{n}$ generate Ricci-flat manifolds on $\left.N^{n+1}\right)$.

Thus, let $(\Sigma, g)$ be any compact $(n-2)$ dimensional Einstein manifold, with Ric $_{g}=(n-3) \cdot g$ and define the warped product metric $\bar{g}$ on $\mathbb{R}^{2} \times \Sigma$ by

$$
\bar{g}=d t^{2}+\frac{4\left(f^{\prime}(t)\right)^{2}}{(n-2)^{2}} d \phi^{2}+f^{2}(t) \cdot g,
$$

where $f$ is the unique function on $[0, \infty)$ such that $f(0)=1, f^{\prime}>0$ and $\left(f^{\prime}\right)^{2}=1-f^{1-n}$. A simple computation shows that $\left(\mathbb{R}^{2} \times \Sigma, \bar{g}\right)$ is complete and Ricci-flat, c.f. [Bes, p.271] and the space-like hypersurface $\mathbb{R}^{+} \times \Sigma$, with metric $d t^{2}+f^{2}(t) \cdot g$, is a solution to the static vacuum equations, with potential $u=f^{\prime}$, (up to a multiplicative constant). Thus the horizon is $\Sigma$ and the solution is smooth up to $\Sigma$ and complete away from $\Sigma$.

This metric is asymptotically conical, i.e. asymptotic to the complete (Euclidean) cone on $(\Sigma, g)$, but is asymptotically flat only in the case that $(\Sigma, g)=S^{n-2}(1)$, corresponding to the $n$-dimensional Schwarzschild metric.

\section{REFERENCES}

[A1] M. Anderson, Scalar curvature, metric degenerations and the static vacuum Einstein equations on 3manifolds I, Geom. \& Funct. Analysis, vol. 9:5, (1999), 855-967; http://www.math.sunysb.edu/ anderson

[A2] M. Anderson, On stationary solutions to the vacuum Einstein equations, to appear in Annales Henri Poincare; http://www.math.sunysb.edu/ anderson .

[A3] M. Anderson, Extrema of curvature functionals on the space of metrics on 3-manifolds, Calc. Var. \& P.D.E., vol. 5, (1997), 199-269.

[AC] M. Anderson and J. Cheeger, Diffeomorphism finiteness for manifolds with Ricci curvature and $L^{n / 2}$-norm of curvature bounded, Geom. \& Funct. Analysis, vol.1:3, (1991), 231-252.

[BW] R. Bach and H. Weyl, Neue Lösungen der Einsteinschen Gravitationsgleichungen, Math. Zeitschrift, vol. 13, (1922), 134-145.

[BKN] S. Bando, A. Kasue and H. Nakajima, On a construction of coordinates at infinity on manifolds with fast curvature decay and maximal volume growth, Inventiones Math., vol. 97, (1989), 313-349.

[Bes] A. Besse, Einstein Manifolds, Springer Verlag, New York, (1987).

[Bn] H. Bondi, Negative mass in general relativity, Rev. Mod. Phys., vol. 29:3, (1957), 423-428. 
[Br] W.B. Bonnor, Physical interpretation of vacuum solutions of Einstein's equations I, Gen. Rel. and Grav., vol. 24:5, (1992), 551-574.

[BM] G. Bunting and A. Massoud-ul-Alam, Non-existence of multiple black holes in asymptotically Euclidean static vacuum space-times, Gen. Rel. and Grav., vol 19, (1987), 147-154.

[CG] J. Cheeger and M. Gromov, Collapsing Riemannian manifolds while keeping their curvature bounded I, II, Jour. Diff. Geom., vol. 23, (1986), 309-346, and vol. 32, (1990), 269-298.

[EK] J. Ehlers and W. Kundt, Exact solutions of the gravitational field equations, in Gravitation: An Introduction to Current Research, Ed. L. Witten, Wiley, N.Y. (1962), 49-101.

[E] A. Einstein, The Meaning of Relativity, $5^{\text {th }}$ Edition, Princeton U. Press, Princeton, (1956).

[El] G.F.R. Ellis, Exact and inexact solutions of the Einstein field equations, in The Renaissance of General Relativity and Cosmology, Ed. G.Ellis et al, Cambridge U. Press, Cambridge, (1993), 20-39.

[ES] G.F.R. Ellis and B.G. Schmidt, Singular space-times, Gen. Rel. and Grav., vol.8:11, (1977), 915-953.

[G] G. Galloway, On the topology of black holes, Comm. Math. Phys., vol 151, (1993), 153-166.

[GH] R. Geroch and J.B. Hartle, Distorted black holes, Jour. Math. Phys, vol. 23:4, (1982), 680-692.

[GT] D. Gilbarg and N. Trudinger, Elliptic Partial Differential Equations of Second Order, $2^{\text {nd }}$ edition, SpringerVerlag, New York, (1983).

[H] W. Hayman, Subharmonic Functions, Academic Press, London, (1976).

[I1] W. Israel, Event horizons in static vacuum space-times, Phys. Review, vol. 164:5, (1967), $1776-1779$.

[I2] W. Israel, Line sources in general relativity, Phys. Review D, vol. 15:4, (1977), 935-941.

[IK] W. Israel and K.A. Khan, Collinear particles and Bondi dipoles in General Relativity, Il Nuovo Cimento, vol. 33:2, (1964), 331-344.

[KN] D. Korotkin and H. Nicolai, A periodic analogue of the Schwarzschild solution, Los Alamos Archive, grqc/9403029, March 1994.

[Kr] D. Kramers, H.Stephani, M. MacCallum, E. Herlt, Exact Solutions of Einstein's Field Equations, Cambridge U. Press, Cambridge, (1980).

[Ku] R. S. Kulkarni, Curvature and metric, Annals of Math., vol. 91, (1970), 311-331.

[L1] A. Lichnerowicz, Theories Relativistes de la Gravitation et de L'Electromagnetisme, Masson, Paris, (1955).

[L2] A. Lichnerowicz, Problemes Globaux en Mechanique Relativiste, Hermann Co., Paris, (1939).

$[\mathrm{Lu}] \quad$ X.-d. Liu, Ball covering on manifolds with non-negative Ricci curvature near infinity, Proc. Symp. Pure Math., Amer. Math. Soc., vol. 54:3, (1993), 459-464.

[MTW] C.W.Misner, K.S. Thorne and J.A. Wheeler, Gravitation, W.H. Freeman, New York, (1973).

[MF] P.M. Morse and H. Feshbach, Methods of Theoretical Physics, McGraw-Hill, New York, (1953).

[P] P.C. Peters, Toroidal black holes? Jour. Math. Phys, vol. 20:7, (1979), 1481-1485.

[Ri] W. Rindler, Essential Relativity, 2nd Edition, Springer Verlag, New York, (1977).

[Ro] D. Robinson, A simple proof of the generalization of Israel's theorem, Gen. Rel. and Grav., vol 8:8, (1977), 695-698.

[SY] R. Schoen and S.T. Yau, Lectures on Differential Geometry, International Press, Cambridge, MA (1994).

[SS] S.M. Scott and P. Szekeres, The Curzon singularity, I: Spatial sections, Gen. Rel. and Grav., vol. 18:6, (1986), 557-570.

[S] P. Szekeres, Multipole particles in equilibrium in general relativity, Phys. Review, vol. 176:1, (1968), 14461450 .

[T] K.S. Thorne, A toroidal solution of the vacuum Einstein field equations, Jour. Math. Phys, vol 16:9, (1975), 1860-1865.

[V] N. Varopoulos, Potential theory and diffusion on Riemannian manifolds, Conf. on Harmonic Analysis in Honor of A. Zygmund, Wadsworth, Belmont, Ca. (1983), 821-837.

[Wd] R. Wald, General Relativity, Univ. of Chicago Press, Chicago (1984).

[Wl] H. Weyl, Zur Gravitationstheorie, Annalen der Physik, vol. 54, (1918), 117-145.

[Wu] H. Wu, An elementary method in the study of non-negative curvature, Acta Math., vol. 142, (1979), 57-78.

July, 1998: revision July, 2000

Department of Mathematics

S.U.N.Y. at Stony Brook

Stony Brook, N.Y. 11794-3651

anderson@math.sunysb.edu 